\title{
CAPM Usando Uma Carteira Sintética do PIB Brasileiro
}

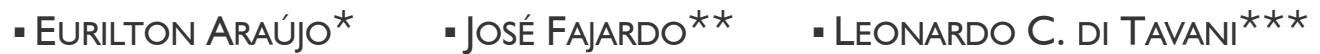

\section{RESUMO}

Uma grande dificuldade de testar o CAPM, como apontado na crítica de Roll, é selecionar uma "proxy" adequada para carteira de mercado. A literatura recente tem buscado alternativas para a construção de uma carteira de mercado, as quais procuram incorporar os efeitos de ativos não transacionados em bolsa, como o capital humano. Este trabalho segue a metodologia proposta por Hou (2002) e se dedica a construir uma carteira de mercado hipotética que paga o PIB como dividendo. $O$ objetivo do artigo é avaliar se esta carteira alternativa constitui uma ampla e legítima "proxy" para a carteira de mercado, testando sua eficiência no sentido média-variância e sua capacidade de explicar o retorno de carteiras de ativos, no contexto do CAPM. Adicionalmente, compara-se o desempenho desta medida alternativa ao obtido pelo retorno do Ibovespa. Os resultados obtidos, especialmente os referentes à versão Black, mostram que a carteira sintética não é eficiente. $O$ retorno do lbovespa, apesar de não satisfazer as condições de validade do CAPM e de eficiência em alguns subperíodos estudados, foi eficiente em muitos deles e também na amostra inteira (1991-2002), sendo, portanto, uma medida mais razoável para a carteira de mercado do que a alternativa proposta por Hou (2002).

Palavras-chave

CAPM, PIB, carteira sintética

\begin{abstract}
One of the difficulties in testing the CAPM, as highlighted in the Roll's critique, is how to select a suitable market proxy. The literature has proposed a number of alternatives to the use of Stock Exchange Indices as a proxy for the Market Portfolio. All of them looked at a broader measure of assets which are not sold at Stock Exchanges. This paper follows Hou (2002) by testing the CAPM through a hypothetical aggregate portfolio that generates GDP as its dividend. The goal of the paper is to evaluate if the proxy proposed by Hou (2002) can be used as a good proxy for the market portfolio in Brazil. In addition, we compare the performance of the synthetic return proposed by Hou (2002) to the Ibovespa return, a very popular proxy for the market portfolio in empirical studies in Brazil. Results, especially the ones related to the Black version of the CAPM, show that the synthetic return measure is not mean-variance efficient for the full sample (199I-2002). In spite of not being able to meet all the requirements imposed by the CAPM model in all sub-samples, the lbovespa return has a better performance compared to the synthetic return. In fact, it is mean-variance efficient for the full sample and for the majority of sub-samples.
\end{abstract}

\section{KEY WORDS}

CAPM, GDP, synthetic portfolio

JEL CLASSIFICATION

$\mathrm{Cl} 2, \mathrm{C} 22, \mathrm{GI} 2$

* Ibmec São Paulo. E-mail: euriltona@ibmec.br.

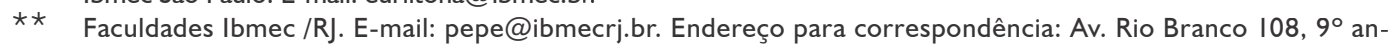
dar - CEP: 20040-00I - Rio de Janeiro, RJ. Agradeço o apoio financeiro do CNPq e da Faperj PRONEX E-26/I7I.193/2003.

$\star \star \star$ Faculdades Ibmec/RJ. E-mail: leocalenda@yahoo.com.

(Recebido em maio de 2004. Aceito para publicação em março de 2006). 


\section{INTRODUÇÃO}

Durante muitos anos o CAPM tem sido um dos pilares da moderna teoria de finanças. No entanto, ainda é questionado se existe evidência empírica deste modelo. Isto é devido ao fato de que para testar o CAPM precisamos usar uma carteira de mercado que não é observável. Daí a necesidade de sempre se usar uma "proxy” para esta carteira. A crítica de Roll (1977) diz basicamente que o CAPM não pode ser testado, uma vez que a carteira de mercado, que deve ser eficiente, não pode ser identificada.

Muitos autores têm se proposto a responder tal crítica. Um caminho óbvio é aumentar a abrangência da carteira de mercado incluindo o maior número de ativos possíveis. Stambaugh (1982) expandiu a carteira de mercado incluindo ações, títulos, automóveis e imóveis. Ele mostra que a inferência não é sensível ao uso de várias "proxies" mais amplas para a carteira de mercado. Campbell (1996) considerou o capital humano explicitamente, uma vez que o retorno do capital humano representa uma grande proporção da renda nacional.

Roll e Ross (1995) e Kandell e Stambaugh (1995) expandem a crítica de Roll, argumentando que a rejeição de uma relação direta entre retorno médio e beta indica a ineficiência da carteira de mercado escolhida e não a teoria do CAPM. Os autores mostram que mesmo carteiras muito diversificadas de todas as ações da amostra irão falhar ao tentar relacionar retorno médio com beta. Além disto, Roll e Ross (1995) derivam a fronteira eficiente de ativos a partir dos Índices de Mercado e demonstram que esta difere da verdadeira fronteira eficiente. Deste modo, esta "proxy" para a carteira de mercado seria ineficiente para determinar o retorno médio a partir do beta. Kandel e Stambough (1995) estenderam esta análise e utilizaram o método de Mínimos Quadrados Geométricos para tentar superar os problemas apontados por Roll e Ross. Basicamente eles mostraram que este método de estimação realmente ajuda, mas apenas para índices de mercado muito próximos de serem eficientes.

Uma alternativa é obter um índice mais representativo, que tenta incorporar um número maior de ativos existentes na Economia, construindo retornos sintéticos baseados em componentes de renda e produto, obtidos a partir das contas nacionais. Baxter et al. (1998) calculam e comparam retornos sintéticos para vários países, todavia não os empregam para testar teorias acerca de apreçamento de ativos.

Mais recentemente, seguindo a linha de Baxter et al. (1998), Hou (2002) construiu uma carteira agregada (conceitualmente mais próxima à carteira de mercado) que paga o Produto Interno Bruto (PIB) Americano como dividendo. Tal construção é, potencialmente, bastante relevante, uma vez que o PIB é o resultado de todos os fatores e atividades da economia, incluindo capital humano, mercado financeiro, imóveis 
e qualquer outro mercado significativo. Portanto, esta carteira agregada seria algo mais próximo à carteira de mercado do ponto de vista conceitual. Todavia, é preciso que a mesma seja eficiente no sentido média-variância e capaz de explicar retornos de ativos. Caso contrário, apesar de seu apelo conceitual de englobar, implicitamente, retornos de ativos não transacionados, tal medida não poderia substituir medidas mais tradicionais, como o retorno do Ibovespa, no caso brasileiro, enquanto carteira de mercado.

Deste modo, a partir da série de dados desta carteira sintética, de acordo com a estrutura do CAPM, tenta-se verificar, por meio de testes econométricos, se a mesma é ou não eficiente no sentido média-variância. A eficiência de uma carteira de mercado baseada no retorno do PIB pode ter importantes implicações. Shiller (1993), por exemplo, argumenta que novos contratos podem ser firmados usando como base o PIB de países distintos, visando à proteção contra riscos macroeconômicos específicos de um dado país.

No Brasil, testes do CAPM usando máxima verossimilhança não foram tão explorados. Contudo, podemos mencionar algumas contribuições: Ribenboim (2002) testa o CAPM na versão Sharpe-Lintner e Black, usando o Ibovespa como carteira de mercado, rejeitando em ambos os casos o CAPM; Silva e Motta (2002) aplicam e testam a versão Black durante o período de 1986 a 2001, dividido em três subperíodos de cinco anos. Empregando o Ibovespa como carteira de mercado, os testes realizados não rejeitam o modelo nos dois últimos subperíodos; por fim, Hagler (2003) testa o CAPM usando vários índices de mercado como proxies para carteira de mercado - a maioria dos testes rejeita o CAPM e aponta a ineficiência de medidas alternativas ao Ibovespa, como os índices IBX 50, IBX 100 e FGV 50.

A proposta deste trabalho é, portanto, testar empiricamente o CAPM tanto para a versão Sharpe-Lintner quanto para a versão Black. Para isto, será adotado o modelo sugerido por Hou (2002), no qual é proposta uma taxa sintética de retorno do PIB como "proxy" para a carteira de mercado; dadas as diferenças entre a economia Brasileira e Americana, algumas modificações serão necessárias. Ainda será testado um índice de mercado bastante usual (Ibovespa) como "proxy" para a carteira de mercado para comparar os resultados obtidos.

O presente trabalho não inova metodologicamente e replica estritamente a metodologia proposta por Hou (2002). O ponto é avaliar a eficiência da carteira construída a partir do retorno sintético do PIB e sua capacidade de explicar retornos de ativos para o Brasil, uma vez que os resultados apresentados por Hou (2002) para a Economia Americana são encorajadores. Com efeito, o desempenho da medida alternativa para os Estados Unidos é bom, porquanto a mesma é, na maioria dos períodos, eficiente, 
com exceção de períodos associados à crise do petróleo nos anos 1970. Assim, é importante avaliar se o desempenho do retorno sintético proposto por Hou (2002) é robusto a especificidades de Economias distintas. Deste modo, dado que a dinâmica da Economia Brasileira é bem diferente da Americana, o presente artigo representa uma evidência acerca da possibilidade de generalização da metodologia de Hou (2002) para economias emergentes. Tendo em vista que os índices de bolsa são, em geral, menos representativos como medida de retorno global de ativos, nos países emergentes comparativamente aos Estados Unidos, a "proxy" de Hou (2002) poderia ser um potencial substituto, enquanto carteira de mercado, para os índices de bolsa de países emergentes, caso a mesma seja eficiente e efetivamente explique o retorno de ativos.

Os resultados obtidos, especialmente os referentes à versão Black, mostram que a carteira sintética não é eficiente. O retorno do Ibovespa, apesar de não satisfazer as condições de validade do CAPM e de eficiência em algumas subamostras estudadas, foi eficiente em muitos casos e também no período inteiro analisado (1991-2002), sendo, portanto, uma medida mais razoável para a carteira de mercado do que a alternativa proposta por Hou (2002).

O artigo é apresentado da seguinte forma: na seção 1 é descrita a economia e formulase a estrutura para a estimação do retorno de uma carteira de mercado hipotética baseada no PIB. Na seção 2 é estimada a taxa sintética de retorno para o PIB Brasileiro. Na seção 3 é testado o CAPM. Na seção 4 apresentam-se os resultados obtidos. Por último, têm-se as conclusões.

\section{O MODELO}

\subsection{A Economia}

O modelo teórico apresentado a seguir é descrito em Cochrane (2001). A apresentação é refeita aqui apenas para tornar o texto do artigo autocontido.

Considere um agente representativo que precisa escolher seu perfil de consumo ao longo do tempo. O agente maximiza o valor descontado de sua utilidade esperada dado por: $E_{0} \sum_{t=0}^{\infty} \beta^{t} U\left(c_{t}\right)$. O agente representativo possui $s_{a t}$ unidades do ativo a no começo do período $t, d_{a t}$ é o dividendo pago pelo ativo $a$, cujo preço na data $t$ é $p_{a t}$ em unidades de consumo. Neste contexto, a restrição orçamentária é: $c_{t}+\sum_{a=1}^{A} p_{a t} s_{a t} \leq \sum_{a=1}^{A}\left(p_{a t}+d_{a t}\right) s_{a t}$, para cada instante de tempo. 
A condição de primeira ordem é dada por:

$$
p_{a t}=E_{t}\left[\beta \frac{u^{\prime}\left(c_{t+1}\right)}{u^{\prime}\left(c_{t}\right)}\left(p_{a t+1}+d_{a t+1}\right)\right]
$$

Definindo o fator de desconto estocástico como $m_{t+1}=\beta \frac{u^{\prime}\left(c_{t+1}\right)}{u^{\prime}\left(c_{t}\right)}$ e o retorno do ativo enquanto $R_{a t+1}=\frac{p_{a t+1}+d_{a t+1}}{p_{a t}}$, podemos escrever a equação fundamental para apreçamento de ativos:

$$
E_{t}\left[m_{t+1} R_{a t+1}\right]=1
$$

Particularmente, este artigo considera o caso em que $A=2$. Temos dois ativos a considerar: um ativo arriscado e um ativo sem risco.

Para o ativo livre de risco, temos: $E_{t}\left[m_{t+1}\right]=\frac{1}{R_{f}}$, uma vez que não existe incerteza sobre o retorno deste ativo em função do estado da Economia.

A equação fundamental $E_{t}\left[m_{t+1} R_{a t+1}\right]=1$ pode ser escrita como:

$$
E_{t}\left(m_{t+1}\right) E_{t}\left(R_{a t+1}\right)+\operatorname{cov}_{t}\left(m_{t+1}, R_{a t+1}\right)=1
$$

Esta última equação pode ser reescrita como:

$$
E_{t}\left(R_{a t+1}\right)-R_{f}=\frac{\operatorname{cov}_{t}\left(\left(R_{a t+1}\right), m_{t+1}\right)}{\operatorname{var}_{t}\left(m_{t+1}\right)}\left[-\frac{\operatorname{var}_{t}\left(m_{t+1}\right)}{E_{t}\left(m_{t+1}\right)}\right]
$$

Supondo que o fator de desconto estocástico possa ser expresso como uma função linear do retorno advindo de uma carteira composta pela riqueza total da Economia, isto é, $m_{t+1}=a+b R_{m t+1}$, a expressão acima fica:

$$
\begin{aligned}
& E_{t}\left(R_{m t+1}\right)-R_{f}=\frac{\operatorname{cov}_{t}\left(R_{m t+1}, a+b R_{m t+1}\right)}{\operatorname{var}_{t}\left(a+b R_{m t+1}\right)}\left[-\frac{\operatorname{var}_{t}\left(m_{t+1}\right)}{E_{t}\left(m_{t+1}\right)}\right] \\
& E_{t}\left(R_{m t+1}\right)-R_{f}=\frac{1}{b}\left[-\frac{\operatorname{var}_{t}\left(m_{t+1}\right)}{E_{t}\left(m_{t+1}\right)}\right]
\end{aligned}
$$


Substituindo o resultado acima na expressão para excesso de retorno de um ativo qualquer, usando $m_{t+1}=a+b R_{m t+1}$, temos:

$$
E_{t}\left(R_{a t+1}\right)-R_{f}=\frac{\operatorname{cov}_{t}\left(R_{a t+1}, R_{m t+1}\right)}{\operatorname{var}_{t}\left(R_{m t+1}\right)}\left[E_{t}\left(R_{m t+1}\right)-R_{f}\right]
$$

Definindo o beta do ativo como $\beta_{a}=\frac{\operatorname{cov}_{t}\left(R_{a t+1}, R_{m t+1}\right)}{\operatorname{var}_{t}\left(R_{m t+1}\right)}$, temos a expressão para o CAPM, que servirá de base para a construção dos testes empíricos implementados neste trabalho, a qual é dada pela equação abaixo:

$$
E_{t}\left(R_{a t+1}\right)-R_{f}=\beta_{a}\left[E_{t}\left(R_{m t+1}\right)-R_{f}\right]
$$

\subsection{Modelo de Retorno Esperado Constante}

A metodologia empírica é integralmente baseada no trabalho de Hou (2002) e será descrita a seguir.

A primeira tarefa para análise é estimar a taxa de retorno para esta carteira de mercado hipotética. Será adotado neste trabalho o modelo de retorno esperado constante apresentado em Campbell e Shiller (1988).

Denotando por $R_{m t}$ a taxa de retorno da carteira de mercado no tempo $t, P_{t}$ o preço da carteira no tempo $t, D_{t}$ o dividendo associado a esta carteira no tempo $t$ (todas as variáveis em termos reais), temos:

$$
R_{m t}=\left(\frac{P_{t}+D_{t}}{P_{t-1}}\right)
$$

A taxa contínua associada ao retorno $R_{m t}$ é definida como $r_{m t}=\log \left(R_{m t}\right)$.

Considerando, por hipótese, que a média de $r_{m t}$, denotado por $\mu_{m}$, é constante, podemos log-linearizar a expressão $R_{m t}=\left(\frac{P_{t}+D_{t}}{P_{t-1}}\right)$, obtendo:

$$
r_{m t}-\mu_{m}=(1-\delta) \sum_{i=0}^{\infty} \delta^{i}\left[E_{t}\left(\log \left(D_{t+i}\right)\right)-E_{t-1}\left(\log \left(D_{t+i}\right)\right)\right]
$$


em que $\delta$ é a taxa de desconto determinada pela razão entre a média do dividendo e a média do preço. Esta equação implica que os componentes não esperados das taxas de retorno são determinados a partir de revisões nas expectativas futuras descontando os dividendos.

A expressão acima, derivada no capítulo 7 de Cambpell, Lo e MacKinlay (1997), é uma maneira alternativa de escrever a equação 7.1.25 (p. 264 do trabalho dos autores supracitados), usando a hipótese de que a média da taxa de retorno contínua é constante, a qual torna nulo o último termo do lado direito desta equação. A equação 7.1.25 (p. 264), está originalmente escrita em termos de variações de dividendos, mas pode ser reescrita em função dos dividendos desde que a equação seja ajustada pelo termo $1-\delta$. A log-linearização encontra-se no apêndice A.

Para uma discussão mais completa, remetemos o leitor ao capítulo 7 de Campbell, Lo e MacKinlay (1997), no qual mais detalhes sobre a álgebra subjacente à expressão (1) podem ser encontrados.

No caso apresentado neste trabalho, $D_{t}=Y_{t}$, em que $Y_{t}$ é o PIB real no tempo $t$. Em outras palavras, a carteira hipotética da economia tem preço $P_{t}$ na data $t$ e gera um fluxo de dividendos no tempo na forma de PIB real. Considerando esta carteira hipotética, pode-se construir uma medida mais ampla para a carteira de mercado, uma vez que o PIB representa a capacidade produtiva do país.

\section{ESTIMANDO A TAXA SINTÉTICA DE RETORNO}

\subsection{Dados}

A série do PIB real foi obtida no IBGE, sendo composta de dados trimestrais para o período de janeiro de 1991 até dezembro de 2002. Os dados foram interpolados utilizando-se o método spline de modo que se obteve a série do PIB real mensal.

Existem, obviamente, medidas alternativas para atividade econômica com freqüência mensal, sendo o índice de produção industrial a medida mais utilizada. Contudo, tal índice não mensura, como o PIB, a atividade econômica de um modo global, concentrando-se apenas no desempenho da indústria, que vem perdendo espaço, mesmo nas economias emergentes como o Brasil, para o setor de serviços. Em suma, o índice de produção industrial não seria uma medida compatível com o escopo da análise deste artigo. Poder-se-ia, ainda, tentar extrair um componente comum de várias séries mensais, como consumo de energia, índice de produção industrial e utilização de bens de 
capital, e empregá-lo como medida de atividade econômica. Como o ponto do artigo é avaliar se a metodologia de Hou (2002) tem bom desempenho numa economia emergente, optamos por implementar a interpolação do PIB, conforme o artigo de Hou (2002). Adicionalmente, no intuito de analisar a robustez dos resultados, todos os testes foram refeitos, usando a série de PIB mensal fornecida pelo Banco Central. ${ }^{1}$ Os resultados são apresentados no apêndice $\mathrm{C}$ e comparados com os resultados discutidos na seção 4 deste artigo. Resumidamente, o resultado básico de que a "proxy" é ineficiente no sentido média-variância se mantém.

Uma vez que está sendo procurada uma carteira de mercado que englobe não só os ativos listados em índices de mercado como também imóveis, o capital humano, bens não-tangíveis, enfim, uma carteira representativa da economia, seria mais indicada a construção da carteira sintética a partir do Produto Interno Líquido (PIL). Isto porque esta variável exclui a depreciação presente no PIB. No entanto, como a depreciação é linear ao longo do tempo, usar PIL ou PIB não terá grande impacto na análise. Assim, será utilizada a variável PIB como na abordagem de Hou (2002).

O próximo passo para a estimação da taxa sintética de retorno será encontrar o modelo mais adequado para especificar o processo do PIB Brasileiro.

\subsection{Modelando o Processo do PIB Brasileiro}

O objetivo desta subseção é modelar o PIB real de acordo com um processo estocástico univariado, que possa ser empregado, juntamente com a aproximação linear obtida em 1.2, no intuito de construir a série sintética de retorno associada a uma carteira hipotética que paga o PIB real como dividendo.

Para utilizar a equação (l) na construção da série sintética de retorno é preciso obter estimativas para $E_{t}\left[\log \left(D_{t+i}\right)\right]$. Para tanto, é preciso especificar o modelo que melhor se adapte ao processo do PIB, denotado por $Y_{t}$, posto que, por definição, a carteira hipotética é caracterizada por $D_{t}=Y_{t}$. Sendo $y_{t}=\log Y_{t}$, podemos tomar a primeira diferença e obter $\Delta y_{t}=y_{t}-y_{t-1}=\log \left(\frac{Y_{t}}{Y_{t-1}}\right)$. Em outras palavras, $\Delta y_{t}$ representa a taxa de crescimento do PIB entre os períodos $t-1$ e $t$.

1 Os autores agradecem a sugestão dessa nova "proxy" mensal de atividade econômica a um parecerista anônimo.

Estud. econ., São Paulo, 36(3): 465-505, jul-set 2006 
FIGURA 1 - HISTOGRAMA DA DIFERENÇA LOGARÍTMICA DO PIB

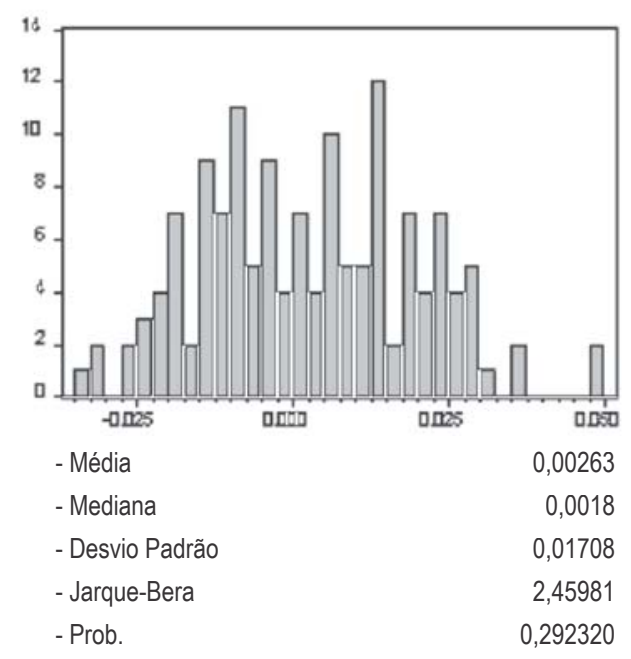

Hou (2002) supõe o seguinte modelo para o processo do PIB real:

$$
\Delta y_{t}=\sum_{i=1}^{p} a i t^{i}+\sum_{j=1}^{q} b_{j} \Delta y_{t-j}+\varsigma t
$$

em que $\varsigma_{t} \sim N\left(0, \sigma_{\varsigma}^{2}\right)$

Os testes ADF para a série do PIB mostram que esta série é estacionária apenas em primeira diferença. Em outras palavras, a série é integrada de ordem 1. Portanto, julga-se adequado trabalhar com a série em primeira diferença. Além disso, foi utilizado o método X12-ARIMA para expurgar o efeito sazonal da série. Após estimar especificações alternativas para o PIB real, e analisando os resultados dos critérios de informação usuais (Schwartz e AIC), bem como a estrutura de correlação serial dos resíduos, ${ }^{2}$ concluiu-se que o processo que melhor descreve esta série é o apresentado abaixo:

$$
\Delta y_{t}=a_{0}+b_{1} \Delta y_{t-1}+b_{3} \Delta y_{t-3}+\varepsilon_{t}
$$

Vale ressaltar que, diferentemente do caso brasileiro, Hou (2002) encontra um processo $\operatorname{AR}(1)$ como melhor especificação para modelagem do PIB americano.

2 Foi feito o teste LM nos resíduos e os resultados indicaram que não há autocorrelação nos resíduos: p-valor 0.7107 (para 4 defasagens) e 0.8850 (para 6 defasagens). 
A tabela a seguir apresenta os parâmetros estimados relativos a esta regressão, com as suas respectivas estatísticas $t$.

$$
\begin{gathered}
\text { TABELA } 1 \text { - RESULTADOS DA ESTIMAÇÃO REFERENTE AO PROCESSO DO } \\
\text { PIB - AR(3) }
\end{gathered}
$$

\begin{tabular}{ccc}
\hline Parâmetros & Estimativas & Estatística $t$ \\
\hline$a_{0}$ & 0,0019 & 2,6926 \\
$b_{1}$ & 0,9457 & 22,1764 \\
$b_{3}$ & $-0,2791$ & $-6,7346$ \\
\hline
\end{tabular}

\subsection{Construindo a Série Sintética de Retorno}

Para a construção do retorno sintético da carteira hipotética associada à atividade econômica global de um país utilizaremos a aproximação log-linear apresentada na seção 1.2, considerando com dividendo da carteira o PIB real.

A equação (2) da seção 2.2 é usada para calcular as expectativas na equação (1) da seção 1.2 , considerando o PIB real como dividendo da carteira hipotética associada à atividade econômica global, ou seja, fazendo $D_{t}=Y_{t}$. Para cada data $t$, usando o processo AR(3), é possível calcular a previsão $i$ passos à frente como sendo $E_{t}\left[\log \left(D_{t+i}\right)\right]$. Efetivamente, a equação (1) da seção 1.2 será empregada para calcular diretamente a série de retorno sintética almejada a menos de sua média, isto é, obtemos diretamente $r_{m t}-\mu_{m}$, sem a necessidade de se obter previamente o preço de cotação para o ativo hipotético que pagaria o PIB real como dividendo.

Para calibrar o $\delta$ foi utilizado o período pós-estabilização, ou seja, de janeiro de 1995 até dezembro de 2002. Considerando a aversão relativa ao risco constante, a utilidade para o agente representativo da economia é descrita conforme a equação abaixo.

É importante notar que essa função-utilidade é amplamente usada em modelos de apreçamento de ativos em tempo discreto.

$$
U=\frac{c_{t}^{1-\sigma}}{1-\sigma}
$$

De acordo com Danthine e Donaldson (2001), uma das implicações da condição de primeira ordem do problema do agente representativo no modelo CCAPM é: 


$$
\overline{\left(1+r_{f}\right)}=\frac{1}{\delta} \frac{1}{\left(\frac{Y_{t+1}}{Y_{t}}\right)^{-\sigma}}
$$

De acordo com as estimativas de Issler e Piqueira (2000), o valor aproximado de $\sigma$ é 2. Segundo os dados utilizados, $\overline{\left(\frac{Y_{t+1}}{Y_{t}}\right)}=1,0015$ e a média da taxa Selic pós-estabilização é de $2 \%$. Isto posto, o valor calibrado para a taxa de desconto $\delta$ é 0.98 .

Por fim, calibramos $\mu_{m}$ de modo a fazer com que esta média coincida com a média da série de crescimento do PIB real mensal.

A tabela a seguir apresenta um comparativo entre as séries das variáveis, $r_{m}$ (taxa sintética de retorno), dysa (diferença logarítmica do PIB excluindo o fator sazonal) e Ibov (Ibovespa).

TABELA 2 - COMPARATIVO ENTRE $r_{m}$, dysa, E Ibov

\begin{tabular}{|c|c|c|c|c|c|}
\hline \multicolumn{2}{|c|}{ Médias } & \multicolumn{2}{|c|}{ Variâncias Relativas } & \multicolumn{2}{|c|}{ Correlações } \\
\hline \multirow[t]{2}{*}{$r_{m}$} & Ibov & $\operatorname{Var}\left(r_{m}\right)$ & $\operatorname{Var}\left(r_{m}\right)$ & $r_{m} \mathrm{e} d y s a$ & $r_{m}$ e Ibov \\
\hline & & $\overline{\operatorname{Var}(d y s a)}$ & $\overline{\operatorname{Var}(I b o v)}$ & & \\
\hline 0,0026 & 0,0241 & 1,825 & 0,002 & 0,457 & 0,0384 \\
\hline
\end{tabular}

Vale explicitar que o retorno do Ibovespa foi calculado como as diferenças entre as cotações do índice em logaritmo entre $t$ e $t-1$. O ideal seria usar as ações que participam do índice para obter o dividendo do índice e calcular o "pay-off" da carteira. Segundo a página da Bolsa de Valores de São Paulo, ${ }^{3}$ a cotação disponibilizada por essas instituições já leva em conta o impacto da distribuição de proventos. Assim, o efeito do pagamento de dividendos é pelo menos parcialmente incorporado na metodologia do índice. Além disso, ao calcularmos o retorno como a primeira diferença em logaritmo da cotação do índice, os nossos resultados ficam comparáveis à maioria dos estudos empíricos já realizados no Brasil, como Ribenboim (2002), Silva e Motta (2002) e Hagler (2003), citados na introdução. Os capítulos 2 e 7 da coletânea organizada por Costa Jr. et al. (2000) também definem retorno do mesmo modo que o definido neste artigo. Em suma, apesar de existir teoricamente definiçóes melhores de retorno, a que empregamos permite comparabilidade com estudos anteriores.

3 www.bovespa.com.br/mercado/ações/índices/ Ibovespa. 
A seguir, apresenta-se um gráfico comparativo entre dysa e $\mathrm{rm}$. A partir da análise da tabela e do gráfico, nota-se que a série de retorno sintético obtida apresenta um padrão mais volátil que o da série de retorno do PIB. Tal série será utilizada como "proxy" do retorno da carteira de mercado para testes do Capital Asset Pricing Model (CAPM). FIGURA 2 - O RETORNO SINTÉTICO E A DIFERENÇA LOGARÍTMICA DO
PIB EXCLUINDO O FATOR SAZONAL

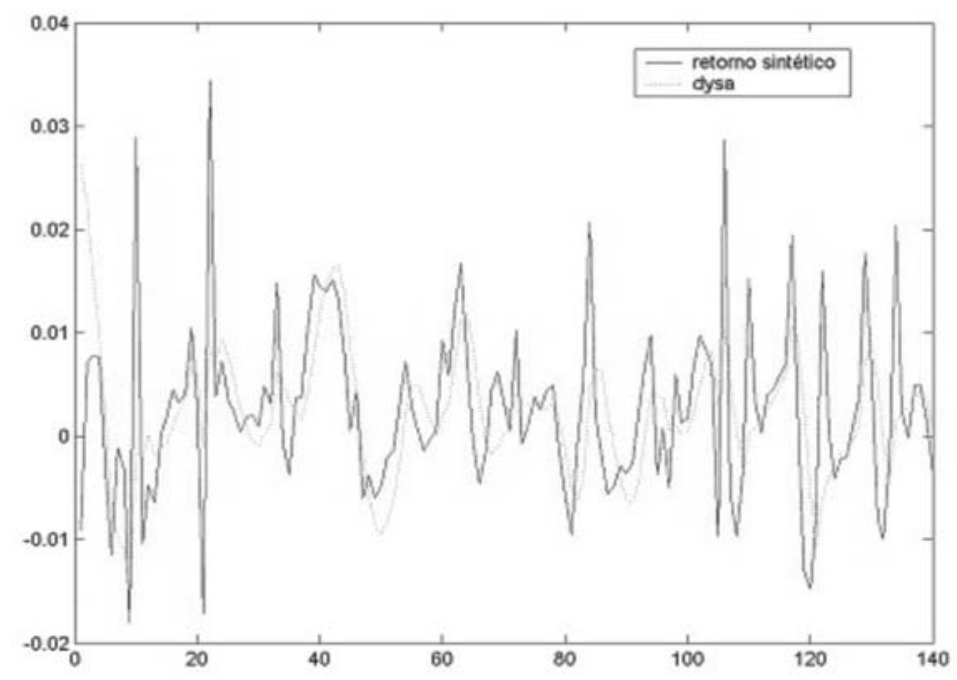

\section{TESTANDO O CAPM}

\subsection{As Duas Versões do Modelo CAPM}

Existem duas versões do modelo CAPM. A versão Sharpe-Lintner parte da hipótese de que todos os investidores podem emprestar e tomar emprestado dinheiro a uma taxa livre de risco $R_{f}$ estabelecendo que, em equilíbrio, a carteira de mercado tem que estar na fronteira eficiente. A partir desta premissa, infere-se que a taxa de retorno de um ativo $i$ em um dado momento $t, R_{i t}$, está linearmente relacionada com o seu $\beta_{i}$ por meio do retorno de mercado $R_{m t}$ conforme:

$$
R_{i t}=R_{f}+\beta_{i}\left[E\left(R_{m}\right)-R_{f}\right]+e_{i t}
$$


Ao introduzir a rentabilidade em excesso de retorno $Z_{i t}=R_{i t}-R_{f}$ e tomar o valor esperado desta expressão, segue que:

$$
E\left(Z_{i t}\right)=\beta_{i}\left[E\left(Z_{m t}\right)\right]
$$

O subscrito m denota a carteira de mercado e a letra maiúscula $\mathrm{Z}$, excesso de retorno em relação a um ativo livre de risco, ou seja, $Z_{m t}=R_{m t}-R_{f}$.

Black relaxa a hipótese de existência de empréstimos sem risco, oferecendo uma nova versão para o modelo:

$$
E\left(R_{i t}-R_{z t}\right)=\alpha+\beta_{i} E\left[\left(R_{m t}\right)-R_{z t}\right]
$$

em que z é a carteira de covariância zero com relação ao mercado.

Ao testar a versão Sharpe-Lintner os requisitos são que o risco deve ser valorizado e que o coeficiente linear desta relação seja zero. Portanto,

$$
H o: \alpha=0 \text { e } E\left(Z_{m t}\right)>0
$$

Para a versão Black o risco também deve ser valorizado, mas o coeficiente linear deve ser maior do que zero. Assim a hipótese nula para teste desta versão deve ser:

$$
H o: \alpha=(i-\beta) \gamma \text { e } E\left(R_{m t}-\gamma\right)>0
$$

em que a letra $i$ denota um vetor da mesma dimensão que o número de ativos com todas os componentes iguais ao número um. E a letra grega $\gamma$ denota o valor esperado do retorno da carteira z, ou seja, $\gamma=E\left(R_{z t}\right)$.

A literatura não aborda testes conjuntos da hipótese de que o risco sistemático é a única variável que explica o retorno e da hipótese de eficiência da carteira de mercado. Portanto, os testes serão realizados de forma independente. Serão feitos para as duas versões, e para cada uma delas será testada tanto a taxa sintética de retorno como "proxy" para a carteira de mercado quanto o Ibovespa. Portanto, teremos quatro cenários para testar empiricamente o CAPM para o Brasil, utilizando a metodologia implementada por Hou (2002):

a) Versão Sharpe-Lintner com a taxa sintética de retorno como carteira de mercado;

b) Versão Sharpe-Lintner com o Ibovespa como carteira de mercado; 
c) Versão Black com a taxa sintética de retorno como carteira de mercado;

d) Versão Black com o Ibovespa como carteira de mercado.

Para a versão Sharpe-Lintner, a hipótese nula $H_{0}: \alpha=0$ é testada de duas formas. Primeiro, por meio de um Teste de Wald. Para tanto, temos duas estatísticas: $J_{0}$, baseada em resultados assintóticos, e $J_{1}$, que implementa uma correção para amostra finita. A segunda maneira de testar essa restrição é por meio de testes de razão de verossimilhança. Tais testes estimam o CAPM duas vezes: uma estimação é irrestrita e a outra incorpora a restrição imposta pela hipótese nula. Novamente, temos as estatísticas de teste $J_{2}$ e $J_{3}$, sendo que está última faz um ajuste para a amostra finita.

Para a versão Black, temos somente testes baseados em razão de verossimilhança para a hipótese nula $H_{0}: \alpha_{i}=\left(1-\beta_{i}\right) \gamma, \forall i$, denotados por $J_{4}$ e $J_{5}$, sendo que este último efetua correção para amostra finita.

A eficiência da carteira de mercado é testada separadamente, via teste $t$, usando a razão de Sharpe, denotada pela letra SR e definida pela razão entre média e desvio padrão amostral do excesso de retorno. Ou seja, a equação que define a razão de Sharpe é

$$
S R=\frac{\overline{Z_{m}}}{D P\left(Z_{m}\right)} .
$$

Detalhes sobre a mecânica da estimação e a distribuição dos estimadores empregados encontram-se no apêndice B.

\subsection{Amostra de Dados para a Estimação do Modelo}

O período da amostra obtida foi de janeiro de 1991 a dezembro de 2002 . No entanto, na construção da taxa sintética de retorno os dados de janeiro a abril de 1991 foram perdidos, devido ao fato de o processo do PIB brasileiro apresentar um comportamento caracterizado por um $\operatorname{AR}(3)$. Neste período foram recolhidos dados de fechamento de 80 ações negociadas na Bolsa de Valores de São Paulo. Tais ações foram alocadas em carteiras conforme seu setor de atuação obtidos no portal Risktech. O retorno das carteiras foi computado a partir do retorno dos ativos independentes seguindo a metodologia de construção de equal-weighted-portfolios, ou seja, o retorno da carteira é obtido a partir da média aritmética dos retornos dos ativos que compõem esta carteira. A razão para a alocação dos ativos em carteiras foi atenuar o risco não-sistemático, aumentando as chances de se captar a relação entre risco sistemático e excessos de retorno. 
A taxa livre de risco estabelecida como parâmetro para a construção das séries de excesso de retorno foi a Selic overnight mensal, obtida no Ipeadata. Alguns autores acreditam que a taxa livre de risco é aquela que o investidor sabe o quanto será remunerado em períodos futuros. Como a Selic é pós-fixada, e em países emergentes a taxa de Juros é alterada freqüentemente, estes autores preferem utilizar taxas pré-fixadas ou CDB. No entanto, neste trabalho a Selic foi escolhida como taxa livre de risco por se tratar da taxa básica de juros da economia servindo de referência para as demais taxas de juros praticadas no mercado. Todas as séries foram deflacionadas pelo IPCA, de modo que todos os testes utilizarão taxas reais.

A seguir, apresentamos uma tabela-resumo dos ativos e seus setores de atuação, bem como a composição das carteiras.

TABELA 3 - COMPOSIÇÃO DAS CARTEIRAS DOS ATIVOS

\begin{tabular}{|c|c|c|}
\hline Classificação & $\begin{array}{l}\text { Quantidade } \\
\text { de ativos }\end{array}$ & Composição da Carteira \\
\hline Alimentos/Bebidas/Fumo & 7 & $\begin{array}{l}\text { Ambev ON / Ambev PN / Avipal ON / Minupar PN / Perdigão PN / Sadia SA PN/ } \\
\text { Souza Cruz ON }\end{array}$ \\
\hline Bancos/Financeiras & 20 & $\begin{array}{l}\text { Alfa Consórcio PNF / Alfa Financeira ON / Alfa Financeira PN / Alfa Holding PNB } \\
\text { / Alfa Investimentos ON / Alfa Investimentos PN / Amazonia ON / Banespa ON / } \\
\text { Banespa PN / Besc PNB / Bradesco ON / Bradesco PN / Brasil ON / Itaubanco } \\
\text { ON / Itaubanco PN / Itausa ON / Itausa PN / Merc S Paulo PN / Sudameris ON } \\
\text { / Unibanco PN }\end{array}$ \\
\hline $\begin{array}{l}\text { Brinquedo/Eletro- } \\
\text { eletrônicos }\end{array}$ & 3 & Bic Caloi PNB / Estrela PN / Weg PN \\
\hline Cimento/Construção & 2 & Eternit ON / Votorantim C P PN \\
\hline Energia/Petroquímica & 16 & $\begin{array}{l}\text { Braskem PNA / Cemig PN / Cesp PN / Eletrobras PNB / F. Cataguazes PNA / } \\
\text { Ipiranga Pet. PN / Ipiranga Ref. PN / Light ON / Paranapanema PN / Paul F Luz } \\
\text { ON / Petrobrás ON / Petrobrás PN / Supergasbras PN / Suzano PN / Trikem PN } \\
\text { / Unipar PNB }\end{array}$ \\
\hline Industria têxtil/Comércio & 4 & Alpargatas PN / Kuala PN / Loj. Americanas PN / Teka PN \\
\hline Mineração/Madeira & 4 & Caemi Metal PN / Duratex PN / Vale do Rio Doce ON / Vale do Rio Doce PNA \\
\hline $\begin{array}{l}\text { Papel Celulose/ } \\
\text { Fertilizantes }\end{array}$ & 5 & Adubos Trevo PN / Aracruz PNB / Fertibras PN / Klabin PN / Ripasa PN \\
\hline $\begin{array}{l}\text { Siderurgia/Metalurgia/ } \\
\text { Autopeças }\end{array}$ & 10 & $\begin{array}{l}\text { Acesita ON / Acesita PN / Belgo Mineira ON / Belgo Mineira PN / Confab PN } \\
\text { / Forjas Taurus PN / Gerdau Met PN / Gerdau PN / Inepar Construções PN / } \\
\text { Metal Leve PN }\end{array}$ \\
\hline $\begin{array}{l}\text { Transporte/ } \\
\text { Telecomunicações }\end{array}$ & 9 & $\begin{array}{l}\text { Embraer PN / lochp-Maxion PN / Marcopolo PN / Randon Par PN / Telemar } \\
\text { Norte Leste ON / Telemar Norte Leste PNE / Telesp Operc ON / Telesp Operc } \\
\text { PN / Varig PN }\end{array}$ \\
\hline
\end{tabular}


Em virtude da implantação do Plano Real em 1994 e da desvalorização cambial em 1999, os testes serão realizados para três períodos distintos devido a uma possível quebra estrutural nestes momentos. É importante ressaltar que as quebras, apesar de terem um forte significado econômico, implicam poucas observações para testes em amostra finita.

Efetivamente, o tamanho pequeno da amostra nos obriga a olhar com cautela para os resultados a serem apresentados na próxima seção. O CAPM envolve uma relação entre retornos esperados de ativos e carteira de mercado. Retornos médios observados só se constituem numa boa aproximação para os retornos esperados caso a amostra tenha um bom tamanho. Desta forma, a rejeição do CAPM e obtenção de razões de Sharpe negativas, inclusive para a carteira de mercado (o que indica ineficiência no sentido média-variância), é bem mais provável num contexto de amostra pequena. No caso brasileiro, há um agravante adicional, que será discutido com detalhes na próxima seção: o alto nível de taxas de juros durante o período analisado.

TABELA 4 - PERÍODOS DE POSSÍVEIS QUEBRAS ESTRUTURAIS

\begin{tabular}{lll}
\hline Período & Amostra & Motivo da Quebra \\
\hline $199101-199407$ & 39 meses & \\
$199408-199901$ & 54 meses & Plano Real \\
$199902-200212$ & 47 meses & Desvalorização Cambial \\
\hline
\end{tabular}

\section{RESULTADOS}

\subsection{Resultado Obtido para a Versão Sharpe-Lintner do Modelo CAPM}

A seguir, têm-se os resultados para os estimadores e estatísticas $t$, utilizando o retorno sintético como "proxy” para a carteira de mercado, assim como as razões $J$. 
TABELA 5 - RESULTADOS - SHARPE-LINTNER UTILIZANDO A TAXA SINTÉTICA DE RETORNO COMO PROXY PARA A CARTEIRA DE MERCADO

\begin{tabular}{|c|c|c|c|c|c|c|c|c|c|c|c|c|}
\hline \multirow{2}{*}{$\begin{array}{l}\text { Proxy Mercado } \\
\text { Taxa de Retorno Sintét. }\end{array}$} & \multicolumn{3}{|c|}{ 1991-1994 } & \multicolumn{3}{|c|}{ 1994-1999 } & \multicolumn{3}{|c|}{ 1999-2002 } & \multicolumn{3}{|c|}{$1991-2002$} \\
\hline & Alfa & $\begin{array}{l}\text { Beta } \\
\text { Irres }\end{array}$ & $\begin{array}{l}\text { Beta } \\
\text { Res }\end{array}$ & Alfa & $\begin{array}{l}\text { Beta } \\
\text { Irres }\end{array}$ & $\begin{array}{l}\text { Beta } \\
\text { Res }\end{array}$ & $f a$ & $\begin{array}{l}\text { Beta } \\
\text { Irres }\end{array}$ & $\begin{array}{c}\text { Beta } \\
\text { Res }\end{array}$ & $f a$ & $\begin{array}{l}\text { Beta } \\
\text { Irres }\end{array}$ & $\begin{array}{l}\text { Beta } \\
\text { Res }\end{array}$ \\
\hline Alim./Bebic & 0354 & $-0,2203$ & 0,6523 & 0,0022 & 1,4564 & 1,3563 & 0,0022 & 1,4564 & 1,0471 & $-0,0065$ & 0,7056 & 0,9225 \\
\hline Desvio Padrão & 0,0273 & 0,9782 & 0,7090 & 0,0214 & 1,2303 & 0,7426 & 0,0214 & 1,2303 & 0,8788 & 0,0109 & 0,5588 & 0,4272 \\
\hline Estatística $t$ & 2947 & $-0,2252$ & 0,9199 & 0,1021 & 1,1838 & 1,8264 & 0,1021 & 1,1838 & 1,1915 & $-0,6020$ & 1,2625 & 2,1596 \\
\hline Bancos/Fina & $-0,0284$ & $-0,8310$ & $-0,1299$ & 0,0066 & 0,7854 & 0,4833 & 0,0066 & 54 & 0,4816 & 58 & $-0,0526$ & 0,13 \\
\hline Desvio Padrão & 0,0244 & 0,8736 & 0,6332 & 0,0197 & 1,1330 & 0,6839 & 0,0197 & 1,1330 & 0,7408 & 0,0097 & 0,4990 & 0,3814 \\
\hline Estatística $t$ & $-1,1649$ & $-0,9512$ & $-0,2052$ & 0,3345 & 0,6932 & 0,7067 & 0,3345 & 0,6932 & 0,6500 & $-0,5925$ & $-0,1055$ & 0,3619 \\
\hline Brinquedo/Elet & $-0,0448$ & $-0,5323$ & 0,5732 & 0,0047 & 3,8600 & 3,6446 & 0,0047 & 3,8600 & 2,0308 & $-0,0104$ & 1,3579 & 1,7027 \\
\hline Desvic & 439 & 1,5719 & 1393 & 0,0261 & 1,5033 & 74 & 0,0261 & 1,5033 & 1,2673 & 0,0159 & 0,8196 & 0,6264 \\
\hline Estat & 0208 & - & 31 & 0,1797 & 2 & 4 , & 97 & 77 & 1,6025 & 24 & 1, & 2,7180 \\
\hline Cimento/Cons & $-0,0341$ & $-0,6022$ & 0,2397 & 0,0073 & 2,4800 & 2,1473 & 0,0073 & 2,4800 & 0,2366 & $-0,0012$ & 0,7903 & 0,8291 \\
\hline Desvi & $0,0<17$ & 0 & 7184 & 0,0245 & 1,4 & 0,8505 & 0,0245 & 1,4091 & 33 & 0,0117 & 04 & 0,4589 \\
\hline Estatística t & $-1,2328$ & $-0,6076$ & 0,3336 & 0,2962 & 1,7600 & 2,5248 & 0,2962 & 1,7600 & 0,2773 & $-0,1002$ & 1,3164 & 1,8068 \\
\hline Energia/Petro & 0,0171 & 1,2135 & ו & $-0,0017$ & 2,0601 & 2,1, & $-0,0017$ & 2,0601 & 1,4791 & 0,0053 & 1,4618 & 1,2867 \\
\hline Desvio $F$ & 0,0373 & 1,3345 & 0,9672 & 0,0234 & 1,3466 & 0,8128 & 0,0234 & 1,3466 & 1,1021 & 0,0135 & 0,6961 & 0,5321 \\
\hline Estatística $t$ & 0,4594 & 0,9093 & 0,8180 & $-0,0721$ & 1,5299 & 2,6299 & $-0,0721$ & 1,5299 & 1,3421 & 0,3900 & 2,0999 & 2,4182 \\
\hline Industria têxt & 0,0103 & 1,7027 & $44 \pi$ & $-0,0062$ & 3,5610 & 3,8471 & $-0,0062$ & 3,5610 & 6,6890 & 0,0279 & 3,7540 & 2,8308 \\
\hline Desvio Padrão & 0,0344 & 1,2323 & 0,8932 & 0,0308 & 1,7708 & 1,0688 & 0,0308 & 1,7708 & 3,1878 & 0,0219 & 1,1232 & 0,8585 \\
\hline Estatística $t$ & 0,3004 & 1,3817 & 1,6208 & $-0,2027$ & 2,0110 & 3,5994 & $-0,2027$ & 2,0110 & 2,0983 & 1,2748 & 3,3423 & 3,2974 \\
\hline Mineração/Mac & $-0,0310$ & $-0,0625$ & 0,7024 & 0,0222 & 3,1522 & 2,1345 & 0,0222 & 3,1522 & $-0,1045$ & 0,0049 & 1,2088 & 1,0448 \\
\hline Desi & 2248 & 0,88 & 0,6430 & 0,0274 & 1,5 & 0,9506 & 0,0274 & 9 & $0, \subseteq$ & 0,0 & 0,6 & 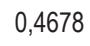 \\
\hline Estatística t & $-1,2516$ & $-0,0705$ & 1,0924 & 0,8105 & 2,0015 & 2,2454 & 0,8105 & 2,0015 & $-0,1149$ & 0,4154 & 1,9749 & 2,2334 \\
\hline Papel & $-0,0503$ & $-1,1310$ & 0,1093 & 0,0121 & 3,2323 & 2,6795 & 0,0121 & 3,2323 & 0 & $-0,0090$ & 0,7072 & 1,0045 \\
\hline Desvio & 0303 & 1,0840 & 0,7857 & 0,0316 & 1,8146 & 1,0953 & 0,0316 & 1,8146 & 1,3336 & 0,0147 & 0,7552 & 0,5773 \\
\hline Estatística t & $-1,6609$ & $-1,0434$ & 0,1392 & 0,3821 & 1,7813 & 2,4464 & 0,3821 & 1,7813 & 0,6855 & $-0,6105$ & 0,9364 & 1,7401 \\
\hline Sider. & $-0,0266$ & $-0,3470$ & 0,3080 & 0,0164 & 3,8475 & 3,0942 & 0,0164 & 3,8475 & 371 & 0,0236 & 2,4329 & 1,6516 \\
\hline Padrão & 0,0349 & 1,2494 & 0,9055 & 0,0234 & 1,3468 & 0,8129 & 0,0234 & 1,3468 & 2,4267 & 0,0180 & 0,9270 & 0,7085 \\
\hline Estatística $t$ & $-0,7610$ & $-0,2777$ & 0,3402 & 0,7016 & 2,8568 & 3,8064 & 0,7016 & 2,8568 & 1,7460 & 1,3073 & 2,6246 & 2,3310 \\
\hline Transporte/Tele & $-0,0075$ & $-0,2973$ & $-0,1130$ & 0,0056 & 2,9058 & 2,6474 & 0,0056 & 2,9058 & 2,0123 & 0,0006 & 1,0162 & 0,9978 \\
\hline drão & 0424 & 1,5186 & 1,1007 & 0,0246 & 1,4134 & 0,8531 & 0,0246 & 1,4134 & ,9028 & 0,0146 & 0,7500 & 0,5733 \\
\hline Estatística $t$ & $-0,1761$ & $-0,1958$ & $-0,1027$ & 0,2293 & 2,0560 & 3,1033 & 0,2293 & 2,0560 & 2,2290 & 0,0380 & 1,3549 & 1,7406 \\
\hline
\end{tabular}

Pode-se observar que todas as estatísticas $t$ referentes à variável $\alpha$ tiveram um valor menor que 1,96 para todos os períodos de teste, o que significa que não se pode 
rejeitar a hipótese de que $\alpha$ seja igual a zero ao nível de significância de $5 \%$ para as carteiras testadas individualmente.

Os testes conjuntos realizados a partir das razões $J$, discutidos detalhadamente no apêndice B, apresentaram o resultado descrito na Tabela 6 .

TABELA 6 - RAZÕES J - SHARPE-LINTNER UTILIZANDO A TAXA SINTÉTICA DE RETORNO COMO PROXY PARA A CARTEIRA DE MERCADO

\begin{tabular}{lcccc}
\hline Proxy mercado - Retorno Sintético & $1991-1994$ & $1994-1999$ & $1999-2002$ & $1991-2002$ \\
$H_{o}: \alpha=0$ & & & & \\
\hline$J_{0}$ & 24,802 & 3,450 & 11,962 & 9,885 \\
$p$-valor & 0,0057 & 0,9688 & 0,2874 & 0,4507 \\
$J_{1}$ & 1,781 & 0,275 & 0,917 & 0,911 \\
$p$-valor & 0,1113 & 0,9836 & 0,5290 & 0,5255 \\
$J_{2}$ & 19,202 & 3,344 & 10,660 & 9,551 \\
$p$-valor & 0,0378 & 0,9721 & 0,3846 & 0,4807 \\
$J_{3}$ & 15,755 & 2,911 & 9,072 & 9,074 \\
$p$-valor & 0,1069 & 0,9835 & 0,5253 & 0,5251 \\
\hline
\end{tabular}

No primeiro período (1991-1994), pode-se observar que o p-valor, da razão $J_{2}$, apresentou um valor abaixo de 0,05 (nível de significância de 5\%). O p-valor da estatística $J_{0}$ também está muito próximo de 0,05 . Isto levaria à rejeição da hipótese de que $\alpha$ seja igual a zero. No entanto, as estatísticas $J_{1} e J_{3}$, que ao contrário de $J_{\mathrm{o}} e J_{2}$ levam em conta o fato de que a amostra é finita, apresentam p-valor acima de 0,05 . Isto implica a não rejeição da hipótese de que $\alpha$ seja igual a zero ao nível de significância de $5 \%$.

No segundo período (1994-1999) todas as razões $J$ implicaram a não rejeição da hipótese de que $\alpha$ seja igual a zero ao nível de significância de $5 \%$, assim como no terceiro período (1999-2002) e no período inteiro sem considerar quebras (1991-2002).

Tal teste de hipótese implica, em grande parte, a aceitação de Ho: $\alpha=0$, ou seja, os excessos de retorno são apenas explicados pelo risco sistemático. No entanto, como apresentado em 3.1, para o CAPM ser válido a carteira de mercado deve ser eficiente no sentido média-variância, ou seja, é preciso testar a hipótese:

$$
\begin{aligned}
& H_{0}: E\left(Z_{m}\right)=0 \\
& H_{a}: E\left(Z_{m}\right)>0
\end{aligned}
$$


Para que a carteira de mercado seja eficiente, a hipótese nula deve ser rejeitada em favor da alternativa $H_{a}$. Contudo, é possível que a alternativa seja rejeitada em favor da hipótese $E\left(Z_{m}\right)<0$. Neste caso, a carteira de mercado também é considerada ineficiente no sentido média-variância. Os resultados obtidos são apresentados a seguir.

\section{TABELA 7 - TESTE DE EFICIENNCIA DA TAXA SINTÉTICA DE RETORNO NA VERSATO SHARPE-LINTNER COMO PROXY PARA A CARTEIRA DE MERCADO}

\begin{tabular}{lcccc}
\hline Proxy mercado - Retorno Sintético & $1991-1994$ & $1994-1999$ & $1999-2002$ & $1991-2002$ \\
$H_{o}: E\left(Z_{m}\right)=0$ & & & & \\
\hline$S R$ & $-0,951$ & $-1,321$ & $-0,518$ & $-0,844$ \\
\hline
\end{tabular}

Na tabela acima, $Z_{m t}=R_{m t}-R_{f}$ é o excesso de retorno da carteira de mercado em relação a um ativo livre de risco e SR denota a razão de Sharpe, definida na seção 3.1.

Nota-se que ao nível de significância de 5\% a hipótese nula não é rejeitada para nenhum período. Além disto, os valores obtidos para SR foram negativos, apesar de não serem estatisticamente significantes ao nível de 5\%. No entanto, é seguro afirmar que a razão não foi positiva em nenhum período, o que implica não se ter a carteira sintética eficiente no sentido média-variância.

Ao utilizar o Ibovespa como "proxy" para a carteira de mercado, obtivemos os seguintes resultados para os estimadores e estatísticas $t$.

No primeiro período (1991-1994), a hipótese de que $\alpha$ é igual a zero foi rejeitada para todas as carteiras ao nível de significância de 5\%. No segundo período (1994-1999), 5 carteiras foram rejeitadas, enquanto que apenas 2 carteiras foram rejeitadas no último período (1999-2002). Ao considerar o período inteiro sem quebras (1991-2002), $60 \%$ das carteiras tiveram a hipótese de que $\alpha$ é igual a zero foi rejeitada ao nível de significância de $5 \%$. 
TABELA 8 - RESULTADOS - SHARPE-LINTNER UTILIZANDO O IBOVESPA COMO PROXY PARA A CARTEIRA DE MERCADO

\begin{tabular}{|c|c|c|c|c|c|c|c|c|c|c|c|c|}
\hline \multirow{2}{*}{$\begin{array}{l}\text { Proxy Mercado } \\
\text { Ibovespa }\end{array}$} & \multicolumn{3}{|c|}{ 1991-1994 } & \multicolumn{3}{|c|}{ 1994-1999 } & \multicolumn{3}{|c|}{ 1999-2002 } & \multicolumn{3}{|c|}{ 1991-2002 } \\
\hline & Alfa & $\begin{array}{l}\text { Beta } \\
\text { Irres }\end{array}$ & $\begin{array}{l}\text { Beta } \\
\text { Res }\end{array}$ & Alfa & $\begin{array}{l}\text { Beta } \\
\text { Irres }\end{array}$ & $\begin{array}{c}\text { Beta } \\
\text { Res }\end{array}$ & Alfa & $\begin{array}{l}\text { Beta } \\
\text { Irres }\end{array}$ & $\begin{array}{l}\text { Beta } \\
\text { Res }\end{array}$ & Ifa & $\begin{array}{l}\text { Beta } \\
\text { Irres }\end{array}$ & $\begin{array}{l}\text { Beta } \\
\text { Res }\end{array}$ \\
\hline Alim. & $-0,0559$ & 0 & 0 & -( & 0 & 01 & 31 & 05 & 71 & $-0,0202$ & 5358 & 5265 \\
\hline Desvio Padrão & 101 & 0,0523 & 0,0510 & 0086 & 0,0687 & 0,0686 & 0,0118 & 0,9898 & 0,8788 & 0,0055 & 0,0395 & 0,0394 \\
\hline Estatísticat & -55151 & 11,0003 & 10,0260 & $-1,7749$ & 8,5235 & 8,5966 & 0,6883 & 1,3745 & 1,1915 & $-3,6708$ & 13,5704 & 13,3607 \\
\hline Bancos/Finar & $-0,0319$ & 0,4517 & 0,4152 & $-0,0019$ & 0,5101 & 0,5106 & 0,0035 & 0,6151 & 0,4816 & $-0,0092$ & 0,4565 & 0,4522 \\
\hline Desvio Padrão & 120 & 0,0622 & 0,0606 & 0,0083 & 0,0664 & 0,0663 & 0,0099 & 0,8344 & 0,7408 & 0,0051 & 0,0368 & 0,0368 \\
\hline Estatística t & $-2,6457$ & 7,2671 & 6,8507 & $-0,2281$ & 7,6825 & 7,6966 & 0,3479 & 0,7372 & 0,6500 & $-1,7923$ & 12,3908 & 12,3005 \\
\hline Brinquedo & $-0,0574$ & 0,5293 & 0,4636 & $-0,0466$ & 0,4617 & 0,4757 & 0,0180 & 2,7249 & 2,0308 & $-0,0317$ & 0,4668 & 0,4521 \\
\hline Desvio F & 201 & 0,1465 & (2) & 0 & 0,1182 & 0,1182 & 0,0170 & 1,4273 & 1,2673 & 0,0110 & 0,0791 & $0,0 T 4$ \\
\hline Estatistica $t$ & $-2,0207$ & 3,6142 & 3,2465 & $-3,1546$ & 3,9046 & 4,0264 & 1,0572 & 1,9091 & 1,6025 & $-2,8673$ & 5,9050 & 5,7313 \\
\hline Cimento/C & $-0,0422$ & 0,4561 & 0,4078 & $-0,0250$ & 0,4605 & 0,4680 & 0,0230 & 1,1263 & 0,2366 & $-0,0150$ & 0,4375 & 0,4305 \\
\hline Desvio Padrão & 152 & 0,0787 & 0,0767 & 0,0131 & 0,1046 & 0,1045 & 0,0114 & 0,9611 & 0,8533 & 0,0074 & 0,0529 & 0,0528 \\
\hline Estati & $-2,76$ & 5,79 & 5 & -1 & 4,40 & 5 & 23 & 1,1 & 0,2 & $-2,0367$ & 8,2728 & 8,1580 \\
\hline Energia/F & $-0,0411$ & 0,8098 & 0,7627 & $-0,0268$ & 0,7235 & 0,7316 & 0,0116 & 1,9265 & 1,4791 & $-0,0198$ & 0,7477 & 0,7385 \\
\hline Desvio Padrão & 130 & 0,0670 & 0,0653 & 0,0077 & 0,0618 & 0,0618 & 0,0148 & 1,2413 & 1,1021 & 0,0058 & 0,0415 & 0,0414 \\
\hline Estat & -3 & 12,0 & 11,6 & 09 & 11, & 11,8 & 0,7835 & 1,5521 & 1,3421 & $-3,4176$ & 18,0072 & $11,0<4$ \\
\hline Ind. têxtil/Con & $-0,0521$ & 0,6889 & 0,6293 & $-0,0528$ & 0,5990 & 0,6149 & 0,0784 & 9,7193 & 6,6890 & $-0,0253$ & 0,6736 & 0,6618 \\
\hline Desvio Padrão & 155 & 0,0801 & 00781 & 0161 & 0, & & 27 & 3,5 & & 5 & 8 & 0,1106 \\
\hline Estatisticat & $-3,3562$ & 8,6052 & 8,0613 & $-3,2162$ & 4,5635 & 4,6881 & 1,8348 & 2,7072 & 2,0983 & $-1,6366$ & 6,0781 & 5,9846 \\
\hline Mine & $-0,0516$ & 0 , & 0 & $-0,0184$ & 0 , & 0 , & 34 & 0 , & -0 & -0 & 54 & 0,4886 \\
\hline Desvio Padrão & 100 & 0,0514 & 0,0501 &, 0131 & 0,1046 & 0,1045 & 0,0122 & 1,0237 & 0,9089 & 0,0072 & 0514 & 0,0513 \\
\hline Estati & $-5,1825$ & 9,8525 & 8,9246 & $-1,4080$ & 6,2829 & 6,3406 & 1,9191 & 0,7808 & -0 & $-2,0475$ & 9,6375 & 9,5248 \\
\hline Papel & $-c$ & 0 & $?$ & -0 & 0,7701 & 0, & 7 & 30 & 0 & -0 & 58 & $T$ \\
\hline Desvio Padrão & 170 & 0,0878 & 0,0856 & 0,0147 & 0,1174 & 0,1173 & 0,0179 & 1,5020 & 1,3336 & 0,0091 & 0,0653 & 0,0652 \\
\hline Estatística t & $-2-2$ & 5 & s & -1 & 6 , & 7 & 32 & 9 & 0 & -2 & 52 & 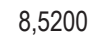 \\
\hline Sider./Metal./Au & $-0,0478$ & 0,6490 & 0,5942 & $-0,0343$ & 0,5473 & 0,5577 & 0,0779 & 7,2479 & 4,2371 & $-0,0125$ & 0,6093 & 0,6035 \\
\hline Desvio Padrão & $0 \cap 167$ & 0,0862 & $0 \cap 841$ & 0,0120 & 0,0962 & 0,0961 & 0,0325 & 2,7330 & 2,4267 & 0,0122 & 0,0873 & 0,0872 \\
\hline Estatística $t$ & $-2,8610$ & 7,5268 & 7,0680 & $-2,8545$ & 5,6902 & 5,8020 & 2,3948 & 2,6520 & 1,7460 & $-1,0213$ & 6,9769 & 6,9253 \\
\hline Transporte/ & $-0,0377$ & 0,8348 & 0,7917 & $-0,0313$ & 0,7177 & 0,7271 & 0,0202 & 2,7943 & 2,0123 & $-0,0189$ & 0,7383 & 0,7295 \\
\hline Desvic & 185 & 0,0953 & 0,0930 & 0,0095 & 0,0763 & 0,0762 & 0,0121 & 1,0167 & 0,9028 & 0,0072 & 0,0512 & 0,0511 \\
\hline Estatistica $t$ & $-2,0388$ & 8,7562 & 8,5161 & $-3,2790$ & 9,4116 & 9,5421 & 1,6719 & 2,7482 & 2,2290 & $-2,6390$ & 14,4182 & 14,2772 \\
\hline
\end{tabular}

Os testes conjuntos realizados a partir das razões $J$ apresentaram o resultado descrito na próxima tabela. 


\section{TABELA 9 - RAZÕES J - SHARPE-LINTNER UTILIZANDO O IBOVESPA} COMO PROXY PARA A CARTEIRA DE MERCADO

\begin{tabular}{lrrrr}
\hline Proxy mercado - Ibovespa & $1991-1994$ & $1994-1999$ & $1999-2002$ & $1991-2002$ \\
$H_{o}: \alpha=0$ & & & & \\
\hline$J_{0}$ & 96,364 & 39,697 & 15,760 & 28,887 \\
$p$-valor & 0,0000 & 0,0000 & 0,1067 & 0,0013 \\
$J_{1}$ & 6,919 & 3,161 & 1,207 & 2,662 \\
$p$-valor & 0,0000 & 0,0041 & 0,3192 & 0,0055 \\
$J_{2}$ & 48,532 & 29,759 & 13,591 & 26,263 \\
$p$-valor & 0,0000 & 0,0009 & 0,1925 & 0,0034 \\
$J_{3}$ & 39,821 & 25,901 & 11,567 & 24,950 \\
$p$-valor & 0,0000 & 0,0039 & 0,3151 & 0,0054 \\
\hline
\end{tabular}

Como esperado, a partir dos resultados dos testes individuais, tanto no primeiro período quanto no segundo, as razões $J_{o}, J_{1}, J_{2} e J_{3}$ apresentaram um p-valor menor que 0,05 , o que significa que a hipótese de que $\alpha$ é igual a zero foi rejeitada ao nível de significância de $5 \%$. Já no terceiro período, que havia apenas duas carteiras em que a hipótese nula tinha sido rejeitada, todas as razóes $J$ apresentaram p-valor acima de 0,05 e, portanto, a hipótese de que $\alpha$ é igual a zero não foi rejeitada. No período inteiro as razões $J_{0} e J_{2}$ apresentaram um p-valor menor que 0,05 , enquanto $J_{1} e J_{3}$ indicaram a não rejeição da hipótese nula.

Como no teste feito para a taxa sintética de retorno, isto implicou que, em grande parte dos períodos, a hipótese do risco sistemático ser a única variável que explica os excessos de retorno foi rejeitada, utilizando o Ibovespa como "proxy" para a carteira de mercado. No entanto, para validar o CAPM em determinado período é também necessário que a carteira de mercado utilizada seja eficiente no sentido média-variância. Assim, a mesma razão será testada para o terceiro período (1999-2002) no caso do Ibovespa. O resultado obtido é apresentado a seguir.

\section{TABELA 10 - TESTE DE EFICIENCIA DO IBOVESPA NA VERSÃO SHARPE- LINTNER COMO PROXY PARA A CARTEIRA DE MERCADO}

\begin{tabular}{lcccc}
\hline $\begin{array}{l}\text { Proxy mercado - Ibovespa } \\
H_{0}: E\left(Z_{m}\right)=0\end{array}$ & $1991-1994$ & $1994-1999$ & $1999-2002$ & $1991-2002$ \\
\hline$S R$ & 0,227 & $-0,038$ & $-0,034$ & 0,065 \\
\hline
\end{tabular}

Nota-se que não se pode rejeitar a hipótese nula ao nível de significância de 5\%. Assim como o retorno sintético, o Ibovespa não se mostrou eficiente no sentido 
média-variância para todas as subamostras. Isto porque, mesmo que a razão SR seja positiva, ela é estatisticamente igual a zero ao nível de significância de 5\%. Todavia, para o período 1991-2002, a razão SR, apesar de não ser significativamente diferente de zero, apresentou um valor positivo. Ou seja, em termos de eficiência, o retorno do Ibovespa apresenta resultados marginalmente superiores ao retorno sintético (SR negativo para todos os períodos).

\subsection{Resultado Obtido para a Versão Black do Modelo CAPM}

Na sequiência, temos os resultados obtidos para a versão Black, utilizando a taxa sintética de retorno como "proxy" para a carteira de mercado.

\section{TABELA 11 - RESULTADOS - BLACK UTILIZANDO A TAXA SINTÉTICA DE RETORNO COMO PROXY PARA A CARTEIRA DE MERCADO}

\begin{tabular}{|c|c|c|c|c|c|c|c|c|c|c|c|c|}
\hline \multirow{2}{*}{$\begin{array}{l}\text { Proxy Mercado } \\
\text { Taxa de Retorno } \\
\text { Sintético }\end{array}$} & \multicolumn{3}{|c|}{$1991-1994$} & \multicolumn{3}{|c|}{ 1994-1999 } & \multicolumn{3}{|c|}{$1999-2002$} & \multicolumn{3}{|c|}{ 1991-2002 } \\
\hline & Alfa & $\begin{array}{l}\text { Beta } \\
\text { Irres }\end{array}$ & $\begin{array}{l}\text { Beta } \\
\text { Res }\end{array}$ & Alfa & $\begin{array}{l}\text { Beta } \\
\text { Irres }\end{array}$ & $\begin{array}{l}\text { Beta } \\
\text { Res }\end{array}$ & Alfa & $\begin{array}{l}\text { Beta } \\
\text { Irres }\end{array}$ & $\begin{array}{l}\text { Beta } \\
\text { Res }\end{array}$ & Alfa & $\begin{array}{l}\text { Beta } \\
\text { Irres }\end{array}$ & $\begin{array}{l}\text { Beta } \\
\text { Res }\end{array}$ \\
\hline Alim./Bebidas/Fumo & 0,0001 & $-0,1413$ & 0,3526 & $-0,0027$ & $-2,4512$ & $-2,3049$ & $-0,0062$ & 1,7907 & 1,4657 & 0,0059 & 1,0946 & 0,7035 \\
\hline Bancos/Financeiras & 0,0109 & $-0,3217$ & $-0,3361$ & 0,0170 & $-2,8487$ & $-2,8787$ & 0,0087 & 1,3355 & 0,4505 & 0,0048 & 1,2938 & 0,8028 \\
\hline Brinquedo/Eletroeletr. & $-0,0091$ & $-1,2349$ & 0,2398 & 0,0088 & $-8,1158$ & $-7,8839$ & $-0,0463$ & 5,3074 & 4,3537 & 0,0061 & 1,9510 & 0,8705 \\
\hline Cimento/Construção & 0,0016 & 0,9475 & 0,8834 & 0,0012 & $-0,6938$ & $-0,6464$ & $-0,0193$ & 3,2753 & 2,4284 & 0,0217 & 1,2562 & $-0,1094$ \\
\hline Energia/Petroquímica & 0,0018 & 0,1158 & 0,4058 & 0,0254 & $-3,6567$ & $-3,7389$ & $-0,0208$ & 2,6665 & 2,4864 & 0,0027 & 2,4937 & 1,1652 \\
\hline Ind. têxti//Comércio & $-0,0091$ & 1,9192 & 2,0115 & 0,0021 & $-0,9788$ & $-0,9299$ & $-0,0550$ & 6,0263 & 4,9767 & 0,0270 & 2,4660 & $-0,1404$ \\
\hline Mineração/Madeira & 0,0026 & 0,9113 & 0,8107 & $-0,0054$ & $-0,9552$ & $-0,8354$ & $-0,0176$ & 4,7616 & 2,4190 & 0,0257 & 0,5158 & $-0,4798$ \\
\hline Papel Celulose/Fertiliz. & $-0,0036$ & 0,3604 & 0,8389 & 0,0070 & $-5,1335$ & $-4,9874$ & $-0,0302$ & 5,2960 & 3,2912 & 0,0084 & 3,0834 & 0,9862 \\
\hline Sider./Metal./Autopeças & 0,0097 & $-0,5800$ & $-0,4168$ & 0,0170 & $-5,6574$ & $-5,5897$ & $-0,0345$ & 5,3193 & 3,5711 & 0,0433 & 1,1398 & $-1,2955$ \\
\hline Transporte/Telecomun. & 0,0023 & 0,2444 & 0,4493 & 0,0326 & $-4,7069$ & $-4,8225$ & $-0,0286$ & 3,9697 & 3,0936 & 0,0050 & 3,1332 & 1,1757 \\
\hline gama estimado & & 0,0080 & & & 0,0036 & & & 0,0141 & & & 0,0147 & \\
\hline
\end{tabular}

A seguir são também apresentados os resultados dos testes baseados nas razões $J$. 
TABELA 12 - RAZÕES J - BLACK UTILIZANDO A TAXA SINTÉTICA DE RETORNO COMO PROXY PARA A CARTEIRA DE MERCADO

\begin{tabular}{lllll}
\hline Proxy mercado - Ibovespa & $1991-1994$ & $1994-1999$ & $1999-2002$ & $1991-2002$ \\
$H_{0}: \alpha=(i-\beta) \gamma$ & & & & \\
\hline$J_{4}$ & 7,474 & 3,229 & 7,070 & 8,669 \\
$p$-valor & 0,5879 & 0,9545 & 0,6298 & 0,4684 \\
$J_{5}$ & 6,133 & 2,810 & 6,017 & 8,236 \\
$p$-valor & 0,7266 & 0,9713 & 0,7382 & 0,5106 \\
$J_{6}$ & 0,592 & 0,269 & 0,593 & 0,826 \\
$p$-valor & 0,8070 & 0,9849 & 0,8091 & 0,6045 \\
\hline
\end{tabular}

Nota-se claramente a não rejeição da hipótese de que $\alpha$ é igual a $(i-\beta) \gamma$ para todos os períodos, bem como para a amostra inteira. No entanto, para validar o CAPM é também necessário testar a hipótese de que a carteira de mercado é eficiente no sentido média-variância. Para verificar se é plausível aceitar esta hipótese, é necessário realizar o teste apresentado abaixo.

$$
\begin{aligned}
& H_{0}: \gamma-\mu_{m}=0 \\
& H_{a}: \gamma-\mu_{m}<0
\end{aligned}
$$

em que $\gamma=E\left(R_{z t}\right)$ e $\mu_{m}=E\left(R_{m t}\right)$. As variáveis $\gamma$ e $\mu_{m}$ denotam, respectivamente, a média da carteira z (carteira de covariância zero com relação ao mercado) e a média da carteira de mercado.

A rejeição da hipótese nula em favor da alternativa $H_{a}$ implicaria eficiência da carteira de mercado testada. Contudo, é possível que a alternativa seja rejeitada em favor da hipótese $\gamma-\mu_{m}>0$. Neste caso, a carteira de mercado também é considerada ineficiente no sentido média-variância.

Os resultados obtidos utilizando a carteira sintética como "proxy" para a carteira de mercado são apresentados a seguir.

\section{TABELA 13 - TESTE DE EFICIÊNCIA DA TAXA SINTÉTICA DE RETORNO NA VERSÃO BLACK COMO PROXY PARA A CARTEIRA DE $M E R C A D O$}

\begin{tabular}{lrrrr}
\hline Proxy mercado - Retorno Sintético & $1991-1994$ & $1994-1999$ & $1999-2002$ & $1991-2002$ \\
$H_{o}: \gamma-\mu_{m}=0$ & & & & \\
\hline$t$ & 0,519 & 3,164 & 2,664 & 1,933 \\
$p$-valor & 0,3018 & 0,0004 & 0,0039 & 0,0266 \\
\hline
\end{tabular}


Nota-se que se rejeita a hipótese de eficiência para todos os períodos. Isto porque, apesar de alguns valores serem estatisticamente iguais a zero ao nível de significância de $5 \%$, todos eles são positivos. Assim, claramente a eficiência desta "proxy" é rejeitada.

Ao testar a versão Black como "proxy" para a carteira de mercado, os seguintes resultados foram obtidos.

\section{TABELA 14 - RESULTADOS - BLACK UTILIZANDO O IBOVESPA COMO PROXY PARA A CARTEIRA DE MERCADO}

\begin{tabular}{|c|c|c|c|c|c|c|c|c|c|c|c|c|}
\hline \multirow{2}{*}{$\begin{array}{l}\text { Proxy Mercado } \\
\text { Ibovespa }\end{array}$} & \multicolumn{3}{|c|}{ 1991-1994 } & \multicolumn{3}{|c|}{ 1994-1999 } & \multicolumn{3}{|c|}{ 1999-2002 } & \multicolumn{3}{|c|}{ 1991-2002 } \\
\hline & Alfa & $\begin{array}{l}\text { Beta } \\
\text { Irres }\end{array}$ & $\begin{array}{l}\text { Beta } \\
\text { Res }\end{array}$ & Alfa & $\begin{array}{l}\text { Beta } \\
\text { Irres }\end{array}$ & $\begin{array}{l}\text { Beta } \\
\text { Res }\end{array}$ & Alfa & $\begin{array}{l}\text { Beta } \\
\text { Irres }\end{array}$ & $\begin{array}{l}\text { Beta } \\
\text { Res }\end{array}$ & Alfa & $\begin{array}{l}\text { Beta } \\
\text { Irres }\end{array}$ & $\begin{array}{l}\text { Beta } \\
\text { Res }\end{array}$ \\
\hline Alim./Bebidas/Fumo & $-0,0467$ & 0,5764 & 0,5517 & $-0,0084$ & 0,5825 & 0,5758 & 0,0065 & 0,4590 & 0,4751 & $-0,0131$ & 0,5311 & 0,5192 \\
\hline Bancos/Financeiras & $-0,0202$ & 0,4567 & 0,5303 & 0,0062 & 0,5104 & 0,5173 & 0,0059 & 0,4706 & 0,4874 & $-0,0011$ & 0,4599 & 0,4720 \\
\hline Brinquedo/Eletroeletr. & $-0,0462$ & 0,5170 & 0,5072 & $-0,0376$ & 0,4511 & 0,4175 & 0,0093 & 0,3662 & 0,3815 & $-0,0232$ & 0,4509 & 0,4224 \\
\hline Cimento/Construção & $-0,0308$ & 0,4648 & 0,5082 & $-0,0160$ & 0,4541 & 0,4406 & 0,0228 & 0,4424 & 0,4251 & $-0,0065$ & 0,4359 & 0,4388 \\
\hline Energia/Petroquímica & $-0,0359$ & 0,7948 & 0,7474 & $-0,0222$ & 0,7189 & 0,6989 & 0,0059 & 0,6783 & 0,6837 & $-0,0157$ & 0,7348 & 0,7128 \\
\hline Industria têxtil/Comércio & $-0,0443$ & 0,6739 & 0,6326 & $-0,0461$ & 0,5928 & 0,5510 & 0,0296 & 0,7728 & 0,7229 & $-0,0196$ & 0,6414 & 0,6146 \\
\hline Mineração/Madeira & $-0,0409$ & 0,5085 & 0,5149 & $-0,0127$ & 0,6502 & 0,6393 & 0,0255 & 0,3117 & 0,2958 & $-0,0069$ & 0,4889 & 0,4895 \\
\hline Papel Celulose/Fertiliz. & $-0,0390$ & 0,4990 & 0,5127 & $-0,0253$ & 0,7646 & 0,7418 & 0,0137 & 0,5277 & 0,5249 & $-0,0163$ & 0,5628 & 0,5441 \\
\hline Sider./Metal./Autopeças & $-0,0398$ & 0,6437 & 0,6215 & $-0,0267$ & 0,5371 & 0,5133 & 0,0431 & 0,6620 & 0,5896 & $-0,0059$ & 0,5832 & 0,5832 \\
\hline Transporte/Telecomun. & $-0,0341$ & 0,8350 & 0,7831 & $-0,0265$ & 0,7107 & 0,6868 & 0,0104 & 0,5387 & 0,5422 & $-0,0147$ & 0,7309 & 0,7108 \\
\hline gama estimado & & $-0,0882$ & & & $-0,0026$ & & & 0,0262 & & & $-0,0142$ & \\
\hline
\end{tabular}

Na seqüência, são apresentadas as razões J de teste.

\section{TABELA 15 - RAZÕES J - BLACK UTILIZANDO O IBOVESPA COMO PROXY PARA A CARTEIRA DE MERCADO}

\begin{tabular}{lrrrr}
\hline Proxy mercado - Ibovespa & $1991-1994$ & $1994-1999$ & $1999-2002$ & $1991-2002$ \\
$H_{o}: \alpha=(i-\beta) \gamma$ & & & & \\
\hline$J_{4}$ & 7,199 & 28,760 & 7,208 & 14,312 \\
$p$-valor & 0,6164 & 0,0007 & 0,6155 & 0,1117 \\
$J_{5}$ & 5,907 & 25,032 & 6,134 & 13,596 \\
$p$-valor & 0,7492 & 0,0029 & 0,7264 & 0,1374 \\
$J_{6}$ & 0,574 & 3,025 & 0,597 & 1,389 \\
$p$-valor & 0,8212 & 0,0055 & 0,8054 & 0,1922 \\
\hline
\end{tabular}


Ao utilizar o Ibovespa como "proxy" para a carteira de mercado, tem-se que, exceto no segundo período, a hipótese de que $\alpha$ é igual a $(i-\beta) \gamma$ é aceita ao nível de significância de $5 \%$. No entanto, para validar o CAPM é ainda necessário fazer outro teste de hipótese análogo ao implementado para a "proxy" retorno sintético, que testa se a carteira de mercado é eficiente no sentido média-variância. A seguir, temos o resultado do teste realizado.

\section{TABELA 16 - TESTE DE EFICIÊNCIA DO IBOVESPA NA VERSÃO BLACK COMO PROXY PARA A CARTEIRA DE MERCADO}

\begin{tabular}{lrrrr}
\hline Proxy mercado - Ibovespa & $1991-1994$ & $1994-1999$ & $1999-2002$ & $1991-2002$ \\
$H_{o}: \gamma$ - $\mu_{m}=0$ & & & & \\
\hline$t$ & $-10,288$ & $-1,305$ & 3,432 & $-4,956$ \\
$p$-valor & 0,0000 & 0,1917 & 0,0002 & 0,0000 \\
\hline
\end{tabular}

Como no segundo período (1994-1999) a hipótese de que os retornos são explicados exclusivamente por um único fator de risco sistemático foi rejeitada, o CAPM não é, portanto, válido, independentemente do resultado acerca da eficiência do índice. No primeiro período (1991-1994), assim como no período inteiro (1991-2002), a hipótese de que o Ibovespa é eficiente no sentido média-variância não pode ser rejeitada. Já no terceiro período (1999-2002), a hipótese de que o Ibovespa é uma carteira de mercado foi rejeitada.

Em suma, para o período inteiro (1991-2002), a versão Sharpe-Lintner rejeita fortemente o retorno do Ibovespa como único fator de risco sistemático, aceitando marginalmente o retorno sintético. Mesmo sendo aceito como único fator de risco sistemático, o retorno sintético não é eficiente. A versão Black aceita marginalmente o retorno sintético como único fator de risco e aceita com mais força ainda o retorno do Ibovespa. Ademais, o retorno do IBOVESPA é eficiente para o período 1991-2002, contrariamente ao retorno sintético, que não é eficiente quando consideramos o valor pontual de $\gamma$.

\subsection{Discussão dos Resultados}

O conceito teórico de utilizar a série de retorno sintético construída a partir do PIB como "proxy" para a carteira de mercado em testes do CAPM é interessante, uma vez que esta carteira de mercado captura toda a produção da economia, incluindo capital humano, bens não tangíveis, imóveis, entre outros. Entretanto, os resultados dos testes indicaram que a carteira sintética não se apresentou eficiente para o período 
estudado. Isto pode ter ocorrido devido ao baixo crescimento do PIB no período ou a uma taxa livre de risco alta.

A série de retorno sintético apresenta a mesma média (0.0026) que a série do PIB excluindo o fator sazonal. A diferença é que a série de retorno sintético apresenta um padrão mais volátil, conforme apresentado na Tabela 3 da seção 2.3. Um baixo crescimento do PIB, portanto, implicaria certamente a construção da série de retorno sintética com média pequena. Hou (2002) encontra uma forte evidência de que o retorno sintético para a economia americana é eficiente no período 1990 a 1995, enquanto existe forte rejeição para o período 1978 a 1989. O período 1990 a 1995 se caracterizou por um grande crescimento real da economia norte-americana, conforme apresentado na Figura 4. Neste contexto, a carteira de retorno sintético se apresentou eficiente. Em contrapartida, o PIB norte-americano no período 1978 a 1989 não apresentou a mesma performance. O governo de Reagan foi marcado por taxas de crescimento real do PIB que oscilaram bastante, variando de -2,0\% em 1982 a 7,3\% em 1984. ${ }^{4}$ A carteira de retorno sintético neste período apresentou uma evidência muito forte de não eficiência.

FIGURA 4 - VARIAÇÃO REAL - PIB EUA PERÍODO 1978-1995

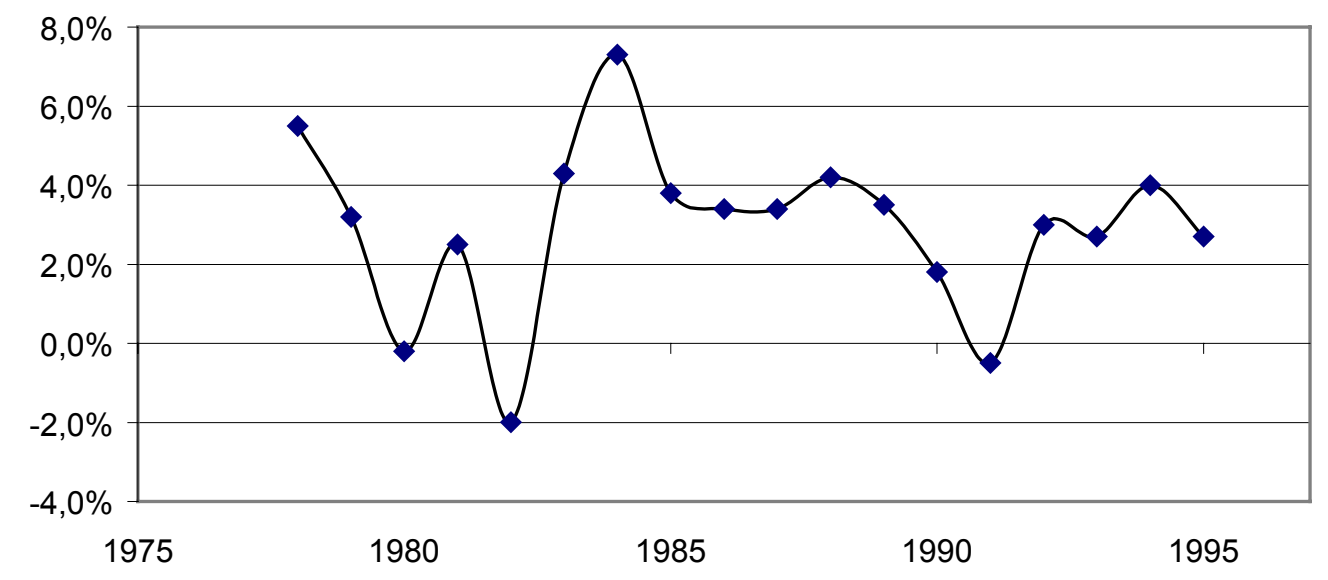

No caso brasileiro, o período 1991-1994 apresentou taxas de crescimento real do PIB com bastante oscilação, enquanto o período 1994-1999 se caracterizou por uma trajetória clara de queda e o período 1999-2002 foi marcado por um baixo crescimento.

4 Fonte: U.S. Department of Commerce, Bureau of Economic Analysis: http://www.bea.doc.gov. 
FIGURA 5 - VARIAÇÃO REAL - PIB BRASILEIRO PERÍODO 1991-2002

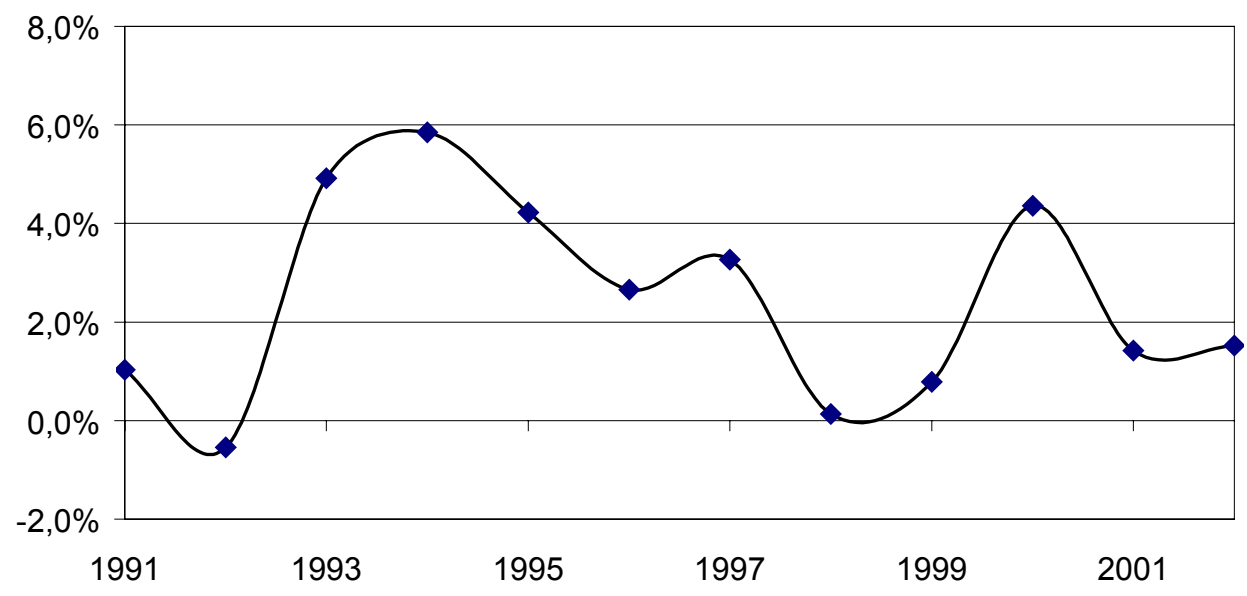

A série de taxa livre de risco utilizada por Hou (2002) para a economia correspondeu a Notas do Tesouro (3 meses). Abaixo é apresentado um gráfico acerca da variação mensal do fechamento da Nota do Tesouro dos Estados Unidos.

FIGURA 6 - VARIAÇÃO PORCENTUAL (\%) - FECHAMENTO TREASURY BILL 30 ANOS

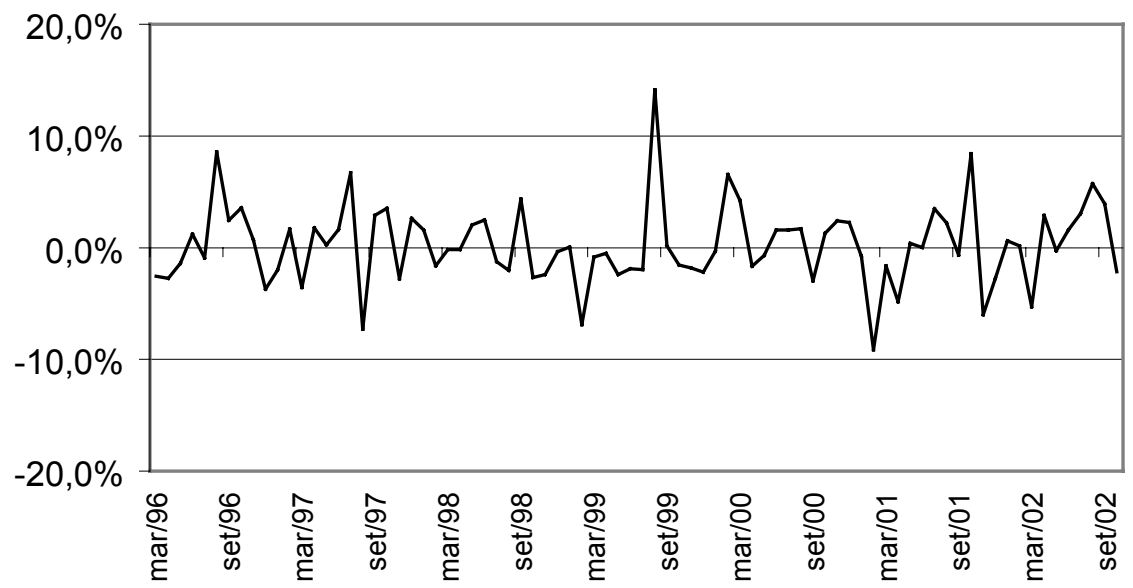

A taxa livre de risco selecionada neste trabalho para o caso brasileiro foi a Selic. Abaixo é apresentado um gráfico da taxa Selic efetiva mensal para o período estudado (1991 a 2002). 


\section{FIGURA 7 - VARIAÇÃO PORCENTUAL (\%) - TAXA SELIC EFETIVA MENSAL}

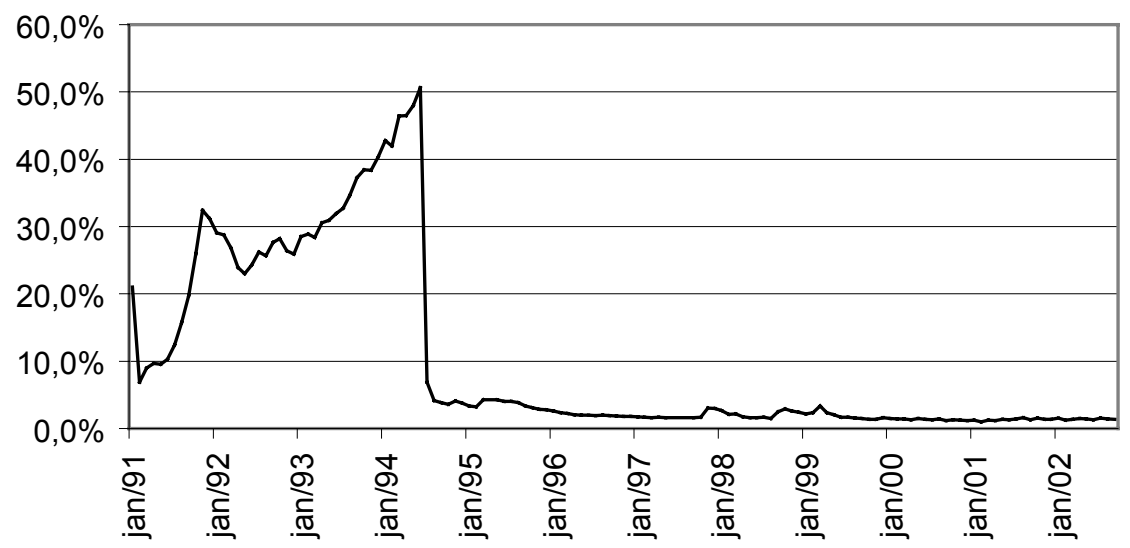

A variância da série das Notas do Tesouro americano é de $0,13 \%$, enquanto a série da Selic efetiva mensal apresentou variância de 1,83\%. O padrão muito volátil da taxa básica de juros da economia brasileira implica o questionamento se esta pode ser considerada realmente uma taxa livre de risco. Este questionamento precisa ser elaborado um pouco mais. ${ }^{5}$ É preciso separar o risco de mercado do risco de "default". Idealmente, o ativo livre de risco deveria ser imune a ambos. Contudo, a literatura convenciona que o ativo livre de risco é imune ao risco de "default". Este parece ser o caso de um título público de curto prazo, como o "T-Bill” de três meses do Tesouro americano. No que tange à taxa Selic, a alta volatilidade não se deve apenas ao risco de mercado, mas também ao risco de "default".

Essa assertiva encontra amparo empírico nos artigos de Gomes e Holland (2003) e Gonçalves et al. (2005). O primeiro artigo mostra a influência da dívida interna e de choques na dívida interna no comportamento da taxa Selic, discutindo a hipótese de que a fragilidade fiscal associada a um alto risco de não pagamento da dívida interna tem impactos não desprezíveis no nível e na volatilidade da Selic. O segundo artigo estuda a conjectura de que a fraca garantia legal, a qual aumenta o risco de "default", explica em certa medida os altos níveis da taxa Selic.

O ponto dos trabalhos citados anteriormente é chamar a atenção para o fato de que o risco de "default" é um componente importante subjacente à alta volatilidade e nível

5 Os autores agradecem ao preciso comentário de um parecerista anônimo, apontando a diferença entre risco de mercado de risco de "default", bem como a relevância da distinção das duas formas de risco na definição de taxa livre de risco. 
da Selic quando comparada à taxa dos Estados Unidos, caracterizada pela virtual ausência de risco de "default".

Portanto, o período 1991 a 2002 se caracterizou por baixo crescimento real do PIB associado a uma "proxy" para a taxa livre de risco muito elevada. Tal combinação implicou forte rejeição da hipótese de eficiência da taxa sintética de retorno, construída a partir do PIB.

\section{CONCLUSÕES}

Pode-se, pois, concluir que a taxa sintética de retorno, apesar de ser conceitualmente mais adequada, não é eficiente no sentido média-variância para todos os períodos em estudo. A versão Black já indicava este fato, apesar da hipótese do retorno ser explicado apenas pelo risco sistemático ter sido aceita para todos os períodos. Quando se impõe a restrição da renda fixa, na versão Sharpe-Lintner, o resultado continua a ser o mesmo. Isto indica que a taxa de retorno sintético que representa dividendos pagos pelo PIB não teve uma boa performance no período 1991 a 2002.

Ao testar o Ibovespa como "proxy" para a carteira de mercado para a versão Black, a hipótese de que o risco sistemático é a única variável que explica o retorno não é rejeitada para o primeiro período (1991-1994), para o terceiro (1999-2002) e para o período inteiro (1991-2002). Ao testar a eficiência da carteira, esta não é rejeitada para o primeiro período e para o período inteiro. No entanto, ao impor a restrição de que existe um ativo sem risco (versão Sharpe-Lintner), a hipótese de que o Ibovespa é eficiente acaba sendo rejeitada para o terceiro período (1999-2002). Este é o único período em que a hipótese de o risco sistemático ser a única variável que explica o retorno não é rejeitada.

Os resultados dos testes relativos à versão Black, por não assumirem um ativo livre de risco, parecem ser mais razoáveis para a Economia brasileira. Segundo esses resultados, o Ibovespa teria sido eficiente e o CAPM teria sido válido para toda a década de 1990.

Portanto, apesar do Ibovespa ter tido uma melhor performance nos testes, não se pode afirmar que este índice foi uma "proxy" robusta para validar o CAPM, uma vez que para alguns períodos, mesmo na versão Black, este índice não foi eficiente.

Comparativamente ao retorno sintético, o retorno do Ibovespa teve um melhor desempenho, especialmente para a amostra inteira. Deste modo, apesar de não ser tão robusto para subperíodos considerados, o retorno do Ibovespa é uma "proxy" mais 
razoável que o retorno sintético, apesar do apelo conceitual deste último. Concluise, pois, que a metodologia proposta por Hou (2002), promissora para os Estados Unidos, não obteve o mesmo sucesso quando aplicada a dados Brasileiros.

\section{REFERENCIAS}

Amihud, Yakov; Bent, Jesper C.; Mendelson, Haim. Futher evidence on the riskreturn relationship. Working Paper, Graduate School of Business, Standard University, 1992.

Baxter, Marianne et al. Synthetic returns on NIPA assets: an international comparison. European Economic Review 42, p. 1141-1172, 1998.

Black, Fischer; Jensen, Michael C.; Scholes, Myron. The capital asset pricing model: some empirical tests. In: Jensen, Michael C. (ed.), Studies in the theory of capital markets. New York: Prager, 1972.

Campbell, J. Understanding risk and return. Journal of Political Economy 104, 1996.

Campbell, John; Lo, Andrew W.; MacKinley, Craig. The econometrics of financial markets. Princeton University Press, 1997.

Campbell, J.; Shiller, R. Stock prices, earnings, and expected dividends. Journal of Finance 43, p. 661-676, 1988.

Cochrane, John. Asset pricing. Princeton University Press, 2001.

Costa Jr. et al. (orgs.). Mercado de capitais: análise empírica no Brasil. Editora Atlas, 2000.

Danthine, Jean-Pierre; Donaldson, John B. Intermediate financial theory. Prentice Hall, 2001.

Fama, Eugene; French, Kenneth R. The cross section of expected stock returns. Journal of Finance 47, p 427-66, 1992.

Fama, Eugene; MacBeth, James. Risk, return and equilibrium: empirical tests. Journal of Political Economy 81, 1973.

Gibbons, M.; Ross, S.; Shaken, J. A test of the efficiency of a given portfolio. Econometrica, 57, p. 1121-1152, 1989.

Gomes, C.; Holland, M. Regra de Taylor e política monetária em condições de endividamento público no Brasil. Economia, v. 4, n. 2, p. 333-361, 2003.

Gonçalves, F. et al. Can jurisdictional uncertainty and capital controls explain the high level of real interest rates in Brazil? Evidence from panel data. Department of Economics, University of California at Berkeley, 2005.

Hagler, Cristina. Testando a eficiência dos indices de ações brasileiros. Departamento de Economia, Ibmec Rio de Janeiro, 2003. 
Hou, Yuanfeng. Testing the CAPM by a synthetic return on GDP as the market return. Economics Department, Yale University, 2002.

Issler, João Victor; Piqueira, Natalia Scotto. Estimating relative risk aversion, the discount rate, and intertemporal elasticity of substitution in consumption for Brazil using three types of utility function. Brazilian Review of Econometrics, 20, 2000.

Jobson, D.; Korkie, R. Potential performance and tests of portfolio efficiency. Journal of Financial Economics, 10, p. 433-466, 1982.

Kandel, Schmuel; Stambaugh, Robert. Portfolio inefficiency and the cross-section of expected returns. Journal of Finance 50, p. 185-224, 1995.

Lintner, John. Security prices, risk and maximal gains from diversification. Journal of Finance 20, 1965.

MacKinley, A. C. On multivariate tests of the CAPM. Journal of Financial Economics, 18, p. 341-372, 1987.

Ribenboim, Guilherme. Teste de modelo CAPM com dados brasileiros. In: Bonomo, Marco Antônio (ed.), Finanças aplicadas ao Brasil. Rio de Janeiro: Editora FGV, 2002.

Roll, Richard; Ross, Stephen. On the cross-sectional relation between expected return and betas. Journal of Finance 50, p. 185-224, 1995.

Shiller, R. Macro markets: creating institutions for managing society's largest economic risks. Oxford University Press, 1993.

Silva, F; Motta, L. Teste do CAPM zero-beta no mercado de capitais brasileiro. Revista de Economia e Administração, v. 1, n. 4, p. 72-88, 2002.

Stambaugh, R. On the exclusion of the assets from tests of the two parameter model. Journal of Financial Economics 10, p. 235-268, 1982.

Tavani, Leonardo C. di. CAPM usando uma carteira sintética do PIB brasileiro. Departamento de Economia, Ibmec Rio de Janeiro, 2003.

\section{APENNDICE A}

Neste apêndice, apresentamos os detalhes da log-linearização da equação:

$$
R_{m t}=\left(\frac{P_{t}+D_{t}}{P_{t-1}}\right)
$$

Em logaritmo, temos: 


$$
\log \left(R_{m t}\right)=\log \left(P_{t}+D_{t}\right)-\log \left(P_{t-1}\right)
$$

A expansão de Taylor de primeira ordem é dada por:

$$
r_{m t} \approx k+\delta p_{t}+(1-\delta) d_{t}-p_{t-1}
$$

em que $\delta=\frac{1}{1+\frac{\bar{D}}{\bar{P}}}$, sendo $\bar{D}$ e $\bar{P}$ valores médios para essas variáveis. Além disso, a constante $k$ é uma função não linear de $\delta$, cuja forma funcional específica é imaterial para a expressão final para o retorno em logaritmo.

Escrevendo a mesma equação um período à frente, temos:

$$
r_{m t+1} \approx k+\delta p_{t+1}+(1-\delta) d_{t+1}-p_{t}
$$

Nas duas equações acima, as variáveis em letra minúscula denotam que as mesmas estão sendo expressas na escala logarítmica.

A equação acima é uma equação a diferenças finitas que pode ser resolvida "para frente". Usando a condição de transversalidade $\lim _{j \rightarrow \infty} \delta^{j} p_{t+j}=0$, a solução pode ser escrita como:

$$
p_{t}=\frac{k}{1-\delta}+\sum_{i=0}^{\infty} \delta^{i}\left[(1-\delta) d_{t+1+i}-r_{m t+1+i}\right]
$$

Calculando a surpresa em termos de retorno, temos:

$$
r_{m t+1}-E_{t}\left(r_{m t+1}\right)=\delta\left[p_{t+1}-E_{t}\left(p_{t+1}\right)\right]+(1-\delta)\left[d_{t+1}-E_{t}\left(d_{t+1}\right)\right]
$$

Usando a solução para $p_{t}$ e o fato de que $p_{t+1}=E_{t+1}\left(p_{t+1}\right)$, podemos escrever a seguinte equação:

$$
r_{m t+1}-E_{t}\left(r_{m t+1}\right)=\delta\left[E_{t+1}\left(A_{t+1}\right)-E_{t}\left(A_{t+1}\right)\right]-\delta\left[E_{t+1}\left(B_{t+1}\right)-E_{t}\left(B_{t+1}\right)\right]+(1-\delta)\left[d_{t+1}-E_{t}\left(d_{t+1}\right)\right]
$$

em que as expressões para $A_{t}$ e $B_{t}$ são, respectivamente:

$$
A_{t}=\sum_{i=0}^{\infty} \delta^{i}\left[(1-\delta) d_{t+1+i}\right] \quad \text { e } \quad B_{t}=\sum_{i=0}^{\infty} \delta^{i} r_{m t+1+i}
$$


Empregando a hipótese de que o retorno médio esperado, em escala logarítmica, é constante, dado pelo valor $\mu_{m}$, temos que $\delta\left[E_{t+1}\left(B_{t+1}\right)-E_{t}\left(B_{t+1}\right)\right]=0$.

A expressão para a surpresa em termos de retorno pode ser escrita como:

$$
r_{m t+1}-E_{t}\left(r_{m t+1}\right)=(1-\delta) d_{t+1}+(1-\delta) E_{t+1}\left[\sum_{i=0}^{\infty} \delta^{i+1} d_{t+2+i}\right]-(1-\delta) E_{t}\left(d_{t+1}\right)-(1-\delta) E_{t}\left[\sum_{i=0}^{\infty} \delta^{i+1} d_{t+2+i}\right]
$$

Finalmente, a expressão pode ser escrita como:

$$
r_{m t+1}-E_{t}\left(r_{m t+1}\right)=(1-\delta) E_{t+1}\left[\sum_{i=0}^{\infty} \delta^{i} d_{t+1+i}\right]-(1-\delta) E_{t}\left[\sum_{i=0}^{\infty} \delta^{i} d_{t+1+i}\right]
$$

Escrevendo a expressão acima um período atrás, temos precisamente a expressão (1) na subseção 1.2, a saber:

$$
r_{m t}-E_{t-1}\left(r_{m t}\right)=(1-\delta) E_{t}\left[\sum_{i=0}^{\infty} \delta^{i} d_{t+i}\right]-(1-\delta) E_{t-1}\left[\sum_{i=0}^{\infty} \delta^{i} d_{t+i}\right]
$$

Usando a mesma notação da subseção 1.2, a equação acima pode ser finalmente escrita como:

$$
r_{m t}-\mu_{m}=(1-\delta) \sum_{i=0}^{\infty} \delta^{i}\left[E_{t}\left(\log \left(D_{t+i}\right)\right)-E_{t-1}\left(\log \left(D_{t+i}\right)\right)\right]
$$

\section{APENNDICE B}

Este apêndice apresenta os detalhes econométricos associados aos Testes do CAPM usando o método da Máxima Verossimilhança.

\section{Testando a Versão Sharpe-Lintner do Modelo CAPM}

Definindo $\mathrm{Z}_{\mathrm{t}}$ como o vetor de excessos de retorno para $\mathrm{N}$ carteiras de acordo com Campbell, Lo e Mackinley (1997), os excessos de retorno poderiam ser descritos utilizando o modelo apresentado abaixo.

$$
\begin{aligned}
& Z_{t}=\alpha+\beta Z_{m t}+e_{t} \\
& E\left[e_{t}\right]=0
\end{aligned}
$$




$$
\begin{aligned}
& E\left[e_{t} e_{t}^{\prime}\right]=\sum \\
& E\left[Z_{m t}\right]=\mu_{m}, E\left[\left(Z_{m t}-\mu_{m}\right)^{2}\right]=\sigma_{m}^{2}, \operatorname{Cov}\left[Z_{m t}, e_{t}\right]=0
\end{aligned}
$$

A versão Sharpe-Lintner implica que todos os elementos do vetor $\alpha$ sejam iguais a zero. Esta conclusão foi derivada das hipóteses de teste do modelo apresentadas na seção 3.1. Se tal condição for satisfeita, a carteira de mercado $m$ seria a carteira tangente da fronteira eficiente.

Neste trabalho será utilizada a abordagem de máxima verossimilhança para encontrar os estimadores do modelo irrestrito. Inicialmente considera-se a função de densidade de probabilidades do excesso de retorno condicionado aos excessos de retorno do mercado. Assumindo que os excessos de retorno para a função de densidade de probabilidade de $\mathrm{Z}_{\mathrm{t}}$ seguem uma distribuição normal, tem-se:

$$
f\left(z_{t} / z_{m t}\right)=(2 \pi)^{\frac{-N}{2}}\left|\sum\right|^{\frac{-1}{2}} \exp \left[-\frac{1}{2}\left(Z_{t}-\alpha-\beta Z_{m t}\right) \sum^{\prime-1}\left(Z_{t}-\alpha-\beta Z_{m t}\right)\right]
$$

A partir da maximização do logaritmo da função de densidade de probabilidade, podem ser encontrados os estimadores de máxima verossimilhança que são os apresentados abaixo.

$$
\begin{aligned}
& \hat{\alpha}=\hat{\mu}-\hat{\beta} \mu_{\hat{m}} \\
& \hat{\beta}=\frac{\sum_{t=1}^{T}\left(z_{t}-\hat{\mu}\right)\left(z_{m t}-\hat{\mu}_{m}\right)}{\sum_{t=1}^{T}\left(z_{m t}-\hat{\mu}_{m}\right)^{2}} \\
& \hat{\Sigma}=\frac{1}{T} \sum_{t=1}^{T}\left(Z_{t}-\hat{\alpha}-\hat{\beta} Z_{m t}\right)\left(Z_{t}-\hat{\alpha}-\hat{\beta} Z_{m t}\right)^{\prime},
\end{aligned}
$$

em que $\hat{\mu}=\frac{1}{T} \sum_{t=1}^{T} Z_{t}$ e $\hat{\mu}_{m}=\frac{1}{T} \sum_{t=1}^{T} Z_{m t}$

As distribuições condicionais dos estimadores $\hat{\alpha}$ e $\hat{\beta}$ seguem uma normal multivariada com médias e variâncias conforme apresentado a seguir. 


$$
\begin{aligned}
& \hat{\alpha} \sim \mathrm{N}\left(\alpha, \frac{1}{T}\left[1+\frac{\hat{\mu}_{m}^{2}}{\hat{\sigma}_{m}^{2}}\right] \Sigma\right) \\
& \hat{\beta} \sim \mathrm{N}\left(\alpha, \frac{1}{T}\left[1+\frac{1}{\hat{\sigma}_{m}^{2}}\right] \Sigma\right) \\
& \hat{\sigma}_{m}^{2}=\frac{1}{T} \sum_{t=1}^{T}\left(Z_{m t}-\hat{\mu}_{m}\right)^{2} \\
& \hat{\mu}_{m}=\frac{1}{T} \sum_{t=1}^{T} Z_{m t}
\end{aligned}
$$

Utilizando os estimadores irrestritos, pode-se formular um teste de Wald para hipótese nula,

$$
\begin{aligned}
& \mathrm{H}_{0}: \alpha=0 ; \\
& \mathrm{H}_{\mathrm{a}}: \alpha \neq 0 .
\end{aligned}
$$

A estatística de teste é:

$$
J_{0}=T\left[1+\frac{\hat{\mu}_{m}^{2}}{\hat{\sigma}_{m}^{2}}\right] \hat{\alpha}^{\prime} \Sigma^{-1} \hat{\alpha} \sim \chi_{N}^{2}
$$

No entanto, neste caso não é necessário recorrer à teoria assintótica para fazer inferências a partir do teste de Wald. A distribuição para amostras finitas apresentada em MacKinlay (1987) e Gibbons, Ross e Shaken (1989), implica uma nova estatística J para o teste.

$$
J_{1}=\frac{(T-N-1)}{N}\left[1+\frac{\hat{\mu}_{m}^{2}}{\hat{\sigma}_{m}^{2}}\right] \hat{\alpha}^{\prime} \Sigma^{-1} \hat{\alpha} \sim \mathrm{F}_{\mathrm{N}, \mathrm{T}-\mathrm{N}-1}
$$

Ambas as estatísticas de teste $J_{0} e J_{l}$ referem-se ao modelo irrestrito. Ao incluir a restrição $\alpha=0$ e resolvendo a partir das condições de primeira ordem, para maximizar a função de Verossimilhança, relativamente a $\beta$ e $\Sigma$, têm-se os seguintes estimadores restritos: 


$$
\begin{aligned}
\hat{\beta}^{*} & =\frac{\sum_{t=1}^{T} Z_{t} Z_{m t}}{\sum_{t=1}^{T} Z_{m t}^{e}} \\
\hat{\Sigma}^{*} & =\frac{1}{T} \sum_{t=1}^{T}\left(Z_{t}-\hat{\beta}^{*}-Z_{m t}\right)\left(Z_{t}-\hat{\beta}^{*}-Z_{m t}\right)^{\prime}
\end{aligned}
$$

As distribuições dos estimadores restritos sobre a hipótese nula são:

$$
\beta^{*} \sim \mathrm{N}\left(\beta, \frac{1}{T}\left[\frac{1}{\hat{\mu}^{2}+\hat{\sigma}^{2}}\right] \Sigma\right)
$$

em que $\hat{\mu}=\frac{1}{T} \sum_{t=1}^{T} Z_{t}$ e $\hat{\sigma}^{2}=\frac{1}{T} \sum_{t=1}^{T}\left(Z_{t}-\hat{\mu}\right)^{2}$

Dados os estimadores de máxima verossimilhança restritos e irrestritos, pode-se testar as restrições impostas pela versão Sharpe-Lintner utilizando o teste da razão de verossimilhança. Este teste é baseado no logaritmo da razão de verossimilhança, que é o valor do logaritmo da função verossimilhança do modelo restrito menos o valor do logaritmo da função de verossimilhança do modelo irrestrito, considerados os valores dos estimadores de máxima verossimilhança. Assim, tem-se:

$$
\begin{aligned}
& L R=L^{*}-L \\
& =-\frac{T}{2}\left[\log \left|\hat{\Sigma}^{*}\right|-\log |\hat{\Sigma}|\right]
\end{aligned}
$$

A partir desta abordagem, pode-se estabelecer a estatística de teste $J_{2}$, que segue uma distribuição qui-quadrado. A estatística é apresentada abaixo:

$$
\begin{aligned}
& J_{2}=-2 L R \\
& =T\left[\log \left|\hat{\Sigma}^{*}\right|-\log |\hat{\Sigma}|\right] \sim \chi_{N}^{2}
\end{aligned}
$$

Utilizando o fato de os estimadores de máxima verossimilhança do modelo restrito poderem ser escritos em termos dos estimadores irrestritos, tem-se: 


$$
\hat{\beta}^{*}=\beta^{*}+\frac{\hat{\mu}_{m}}{\hat{\mu}_{m}^{2}+\hat{\sigma}_{m}^{2}} \hat{\alpha}
$$

Ao inserir esta relação para a matriz de variância e covariância do modelo restrito $\left(\hat{\Sigma}^{*}\right)$, e fazendo algumas operações matemáticas, pode-se concluir que:

$$
L R=-\frac{T}{2} \log \left[\left(\frac{\hat{\sigma}_{m}^{2}}{\hat{\mu}_{m}^{2}+\hat{\sigma}_{m}^{2}}\right) \hat{\alpha}^{\prime} \Sigma^{-1} \hat{\alpha}+1\right]
$$

E, portanto,

$$
J_{2}=T \log \left[\left(\frac{\hat{\sigma}_{m}^{2}}{\hat{\mu}_{m}^{2}+\hat{\sigma}_{m}^{2}}\right) \hat{\alpha}^{\prime} \Sigma^{-1} \hat{\alpha}+1\right]
$$

Para $J_{1}$ tem-se:

$J_{1}=\frac{(T-N-1)}{N}\left(\exp \left[\frac{J_{2}}{T}\right]-1\right)$, que é uma transformação monotônica de $J_{2}$.

Jobson e Korkie (1982) sugerem um ajuste para $J_{2}$ que tem propriedades mais adequadas para amostras finitas.

$$
\begin{aligned}
& J_{3}=\frac{\left(T-\frac{N}{2}-2\right)}{T} J_{2} \\
& =\left(T-\frac{N}{2}-2\right)\left[\log \left|\hat{\Sigma}^{*}\right|-\log |\hat{\Sigma}|\right] \sim \chi_{N}^{2}
\end{aligned}
$$

Portanto, para realizar os testes da versão Sharpe-Lintner foram calculados os estimadores a partir da amostra de dados e da estruturação das carteiras e em seguida foram calculadas as estatísticas $J_{0}, J_{1}, J_{2}$ e $J_{3}$. Estes resultados são apresentados na seção 4.1 para o período inteiro (1991-2002) e para os subperíodos: 1991-1994, 1994-1999 e 1999-2002, bem como para as duas alternativas usadas como "proxy" para a carteira de mercado.

Os testes $J_{1}$ e $J_{3}$ são testes semelhantes a $J_{0} J_{2}$, respectivamente. No entanto, tais testes são ajustados para as propriedades de amostra finita. Como ressaltado anteriormen- 
te, as amostras para os testes são pequenas devido às quebras estruturais ocorridas no período. Assim, para diminuir as chances de utilização de uma amostra pequena implicar precisão dos testes é mais adequada a utilização de $J_{l}$ e $J_{3}$.

\section{Testando a Versão Black do Modelo CAPM}

Conforme apresentado em 3.1, Black relaxa a hipótese de existência de empréstimos sem risco. Assim, o empréstimo livre de risco é representado pela carteira de covariância zero com o mercado $(Z)$. Deste modo o retorno esperado de $i$ é descrito por:

$$
E\left(R_{i t}\right)=E\left(R_{z t}\right)+\beta_{i}\left[E\left(R_{m t}\right)-E\left(R_{z t}\right)\right]=E\left(R_{z t}\right)\left(1-\beta_{i}\right)+\beta_{i} E\left(R_{m t}\right)
$$

Deste modo, a versão Black impõe a seguinte restrição não-linear:

$\alpha=\gamma(i-\beta)$, em que $\gamma=E\left(R_{z t}\right)$ e $\alpha$ e $\beta$ são vetores com componentes de $\alpha_{\mathrm{i}}$ e $\beta_{\mathrm{i}}$.

Então, a questão que se coloca é saber quais são os estimadores para $\alpha$ e $\beta$. Os estimadores de máxima-verossimilhança do modelo irrestrito são os mesmos derivados para o modelo Sharpe-Lintner, exceto pelo fato que neste caso temos Retornos Reais (R) e não excessos de Retorno.

$$
\begin{aligned}
& \hat{\alpha}=\hat{\mu}-\hat{\beta} \mu_{\hat{m}} \\
& \hat{\beta}=\frac{\sum_{t=1}^{T}\left(R_{t}-\hat{\mu}\right)\left(R_{m t}-\hat{\mu}_{m}\right)}{\sum_{t=1}^{T}\left(R_{m t}-\hat{\mu}_{m}\right)^{2}} \\
& \hat{\Sigma}=\frac{1}{T} \sum_{t=1}^{T}\left(R_{t}-\hat{\alpha}-\hat{\beta} R_{m t}\right)\left(R_{t}-\hat{\alpha}-\hat{\beta} R_{m t}\right)^{\prime},
\end{aligned}
$$

em que $\hat{\mu}=\frac{1}{T} \sum_{t=1}^{T} R_{t}$ e $\hat{\mu}_{m}=\frac{1}{T} \sum_{t=1}^{T} R_{m t}$

As distribuições condicionais dos estimadores $\hat{\alpha}$ e $\hat{\beta}$ seguem uma normal multivariada com médias e variâncias conforme apresentado a seguir.

$$
\hat{\alpha} \sim \mathrm{N}\left(\alpha, \frac{1}{T}\left[1+\frac{\hat{\mu}_{m}^{2}}{\hat{\sigma}_{m}^{2}}\right] \Sigma\right)
$$




$$
\begin{aligned}
& \hat{\beta} \sim \mathrm{N}\left(\alpha, \frac{1}{T}\left[1+\frac{1}{\hat{\sigma}_{m}^{2}}\right] \sum\right) \\
& \hat{\sigma}_{m}^{2}=\frac{1}{T} \sum_{t=1}^{T}\left(\hat{R}_{m t}-\hat{\mu}_{m}\right)^{2} \\
& \hat{\mu}_{m}=\frac{1}{T} \sum_{t=1}^{T} R_{m t}
\end{aligned}
$$

A covariância de $\hat{\alpha}$ e $\hat{\beta}$ é:

$$
\operatorname{Cov}[\hat{\alpha}, \hat{\beta}]=-\left[\frac{\hat{\mu}}{\hat{\sigma}_{m}^{2}}\right] \Sigma
$$

Para o modelo restrito, o logaritmo da função de densidade de probabilidade vista em função dos parâmetros desconhecidos, $\alpha, \beta$, e $\Sigma$ é:

$$
(\alpha, \beta, \Sigma)=-\frac{N T}{2} \log (2 \pi)-\frac{T}{2} \log |\Sigma|-\frac{1}{2}\left(R_{t}-\gamma(1-\beta)-\beta R_{m t}\right) \sum^{\prime-1}\left(\left(R_{t}-\gamma(1-\beta)-\beta R_{m t}\right)\right)
$$

Diferenciando com respeito a $\alpha, \beta$, e $\Sigma$ e igualando as equações a zero, encontram-se os seguintes estimadores de máxima-verossimilhança para os estimadores restritos.

$$
\begin{aligned}
& \hat{\beta}=\frac{\sum_{t=1}^{T}\left(R_{t}-\gamma i\right)\left(R_{m t}-\gamma\right)}{\sum_{t=1}^{T}\left(R_{m t}-\gamma\right)^{2}} \\
& \hat{\Sigma}^{*}=\frac{1}{T} \sum_{t=1}^{T}\left(R_{t}-\gamma\left(i-\hat{\beta}^{*}\right)-\hat{\beta}^{*} R_{m t}\right)\left(R_{t}-\gamma\left(i-\hat{\beta}^{*}\right)-\hat{\beta}^{*} R_{m t}\right)^{\prime},
\end{aligned}
$$

Dados ambos os estimadores do modelo irrestrito e restrito, pode-se construir um teste de hipótese cujas hipóteses nula e alternativa são:

$$
\begin{aligned}
& \mathrm{H}_{0}: \alpha=(\mathrm{i}-\beta) \gamma \\
& \mathrm{H}_{\mathrm{a}}: \alpha \neq(\mathrm{i}-\beta) \gamma
\end{aligned}
$$

Uma razão de teste análoga a $J_{2}$ para a versão Sharpe-Lintner pode ser construída do seguinte modo: 


$$
J_{4}=T\left[\log \left|\hat{\Sigma}^{*}\right|-\log |\hat{\Sigma}|\right] \sim \chi_{N-1}^{2}
$$

Pode-se ainda ajustar $J_{4}$ para melhorar as propriedades do teste com amostras finitas. Definido $J_{5}$ como a estatística ajustada tem-se:

$$
J_{5}=\left(T-\frac{N}{2}-2\right)\left[\log \left|\hat{\Sigma}^{*}\right|-\log |\hat{\Sigma}|\right] \sim \chi_{N-1}^{2}
$$

\section{APENNDICE C}

Este apêndice apresenta os resultados dos testes do CAPM usando a série de PIB mensal calculada pelo Departamento Econômico do Banco Central como uma "pro$x y$ ” alternativa para a atividade econômica, na construção da taxa sintética de retorno de uma carteira hipotética que pagaria o PIB como dividendo. Esta série em termos nominais pode ser coletada na página do Banco Central do Brasil (www.bcb.br). A série foi posteriormente deflacionada pelo IPCA.

O período de análise considerado é o mesmo estudado no corpo do texto. A especificação de um processo para a série de PIB real mensal seguem os passos já descritos na seção 2.2. do artigo.

Objetivando tornar o texto conciso, apresentaremos os resultados dos testes J para as versões Sharp-Lintner e Black, bem como os resultados dos testes de eficiência desta nova "proxy" para o retorno da carteira de mercado.

\section{TABELA 17 - RAZÕES J - SHARPE-LINTNER UTILIZANDO A NOVA TAXA SINTÉTICA DE RETORNO COMO PROXY PARA A CARTEIRA DE MERCADO}

\begin{tabular}{lrrrr}
\hline Proxy mercado - Retorno Sintético & $1991-1994$ & $1994-1999$ & $1999-2002$ & $1991-2002$ \\
$H_{0}: \alpha=0$ & & & & \\
\hline$J_{0}$ & 13,374 & 22,39 & 13,21 & 10,74 \\
$p$-valor & 0,2035 & 0,0132 & 0,2117 & 0,3782 \\
$J_{1}$ & 0,960 & 1,784 & 1,012 & 0,989 \\
$p$-valor & 0,4974 & 0,0930 & 0,4521 & 0,4559 \\
$J_{2}$ & 11,499 & 18,73 & 11,648 & 10,35 \\
$p$-valor & 0,0320 & 0,0438 & 0,3010 & 0,4106 \\
$J_{3}$ & 9,436 & 16,31 & 9,913 & 9,829 \\
$p$-valor & 0,1069 & 0,0912 & 0,4481 & 0,4555 \\
\hline
\end{tabular}


TABELA 18 - TESTE DE EFICIÊNCIA DA NOVA TAXA SINTÉTICA DE RETORNO NA VERSÃO SHARPE-LINTNER COMO PROXY PARA A CARTEIRA DE MERCADO

\begin{tabular}{lcccc}
\hline Proxy mercado - Retorno Sintético & $1991-1994$ & $1994-1999$ & $1999-2002$ & $1991-2002$ \\
$H_{o}: E\left(Z_{m}\right)=0$ & & & & \\
\hline$S R$ & $-0,331$ & $-0,374$ & $-0,201$ & $-0,298$ \\
\hline
\end{tabular}

TABELA 19 - RAZÕES J - BLACK UTILIZANDO A NOVA TAXA SINTÉTICA DE RETORNO COMO PROXY PARA A CARTEIRA DE MERCADO

\begin{tabular}{lrrrr}
\hline Proxy mercado - Ibovespa & $1991-1994$ & $1994-1999$ & $1999-2002$ & $1991-2002$ \\
$H_{0}: \alpha=(i-\beta) \gamma$ & & & \\
\hline$J_{4}$ & 7,769 & 18,81 & 11,19 & 9,318 \\
$p$-valor & 0,5576 & 0,0287 & 0,2630 & 0,4080 \\
$J_{5}$ & 6,375 & 16,20 & 9,522 & 8,852 \\
$p$-valor & 0,7019 & 0,0628 & 0,3906 & 0,4510 \\
$J_{6}$ & 0,617 & 1,781 & 0,971 & 0,888 \\
$p$-valor & 0,7862 & 0,0936 & 0,4854 & 0,5464 \\
\hline
\end{tabular}

TABELA 20 - TESTE DE EFICIÊNCIA DA NOVA TAXA SINTÉTICA DE RETORNO NA VERSÃO BLACK COMO PROXY PARA A CARTEIRA DE MERCADO

\begin{tabular}{lrrrr}
\hline Proxy mercado - Retorno Sintético & $1991-1994$ & $1994-1999$ & $1999-2002$ & $1991-2002$ \\
$H_{o}: \gamma-\mu_{m}=0$ & & & & \\
\hline$t$ & $-0,142$ & 3,549 & 1,602 & 1,002 \\
$p$-valor & 0,8873 & 0,0004 & 0,1092 & 0,3166 \\
\hline
\end{tabular}

As conclusões obtidas com o uso da nova "proxy" para a taxa sintética de retorno, construída a partir da série de PIB mensal do Banco Central, são similares às conclusões apresentadas na seção 4, concernentes aos testes do CAPM quando empregamos a taxa sintética, construída via procedimento de Hou (2002). Em resumo, os testes J, em sua maioria, não rejeitam o CAPM, tanto para a versão Sharp-Lintner quanto para a versão Black. Todavia, os testes de eficiência apontam para o fato de que a "proxy" usada para o retorno da carteira de mercado não é eficiente no sentido média-variância. Em suma, os resultados apresentados no corpo do texto são robustos a uma mensuração alternativa do PIB mensal. 